ITEP-TH-19/20

IITP-TH-14/20

\title{
Löwner equations and reductions of dispersionless hierarchies
}

\author{
V. Akhmedova* \\ T. Takebe ${ }^{\dagger}$ \\ A. Zabrodin $\ddagger$ \\ October 2020
}

\begin{abstract}
The equations of Löwner type can be derived in two very different contexts: one of them is complex analysis and the theory of parametric conformal maps and the other one is the theory of integrable systems. In this paper we compare the both approaches. After recalling the derivation of Löwner equations based on complex analysis we review one- and multi-variable reductions of dispersionless integrable hierarhies (dKP, dBKP, dToda, and dDKP). The one-vaiable reductions are described by solutions of different versions of Löwner equation: chordal (rational) for dKP, quadrant for dBKP, radial (trigonometric) for dToda and elliptic for DKP. We also discuss multi-variable reductions which are given by a system of Löwner equations supplemented by a system of partial differential equations of hydrodynamic type. The solvability of the hydrodynamic type system can be proved by means of the generalized hodograph method.
\end{abstract}

\section{Contents}

\section{Introduction}

*Kharkevich Institute for Information Transmission Problems, Bolshoy Karetny per. 19, build.1, Moscow, 127051, Russia, e-mail: valeria-58@yandex.ru

$\dagger$ Faculty of Mathematics, National Research University Higher School of Ecnomics, Russian Federation, Usacheva str., 6, Moscow, 119048; HSE-Skoltech International Laboratory of Representation Theory and Mathematical Physics, Usacheva str., 6, Moscow, 119048, Russia, e-mail: ttakebe@hse.ru

${ }^{\ddagger}$ Skolkovo Institute of Science and Technology, 143026, Moscow, Russia and Institute of Biochemical Physics, Kosygina str. 4, 119334, Moscow, Russia and ITEP NRC KI, 25 B.Cheremushkinskaya, Moscow 117218, Russia, e-mail: zabrodin@itep.ru 
2 Löwner equations

2.1 Chordal Löwner equation . . . . . . . . . . . . . . . . . . 4 4

2.2 Quadrant Löwner equation . . . . . . . . . . . . . . . . . . . . 8 8

2.3 Radial Löwner equation . . . . . . . . . . . . . . . . . . . . . 9 9

2.4 Komatu-Löwner equation . . . . . . . . . . . . . . . . . 13

3 One-variable reductions of integrable hierarchies $\quad 17$

3.1 One-variable reductions of the dKP hierarchy . . . . . . . . . . . . 17

3.1.1 The dKP hierarchy ................... 17

3.1 .2 The reduction . . . . . . . . . . . . . . . . 19

3.2 One-variable reductions of the dBKP hierarchy . . . . . . . . . . . 21

3.2.1 The dBKP hierarchy . . . . . . . . . . . . . . . . . . . . 21

3.2 .2 The reduction . . . . . . . . . . . . . . . 22

3.3 One-variable reductions of the dToda hierarchy . . . . . . . . . . . 23

3.3.1 The dToda hierarchy . . . . . . . . . . . . . . . 23

3.3 .2 The reduction . . . . . . . . . . . . . 26

3.4 One-variable reductions of the dDKP hierarchy . . . . . . . . . . . . . 28

3.4.1 Algebraic form of the dDKP hierarchy . . . . . . . . . . . . 29

3.4.2 Elliptic form of the dDKP hierarchy . . . . . . . . . . 30

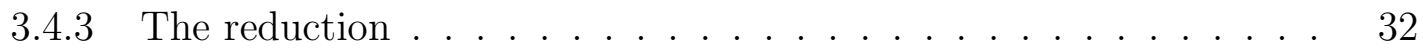

4 Multivariable reductions 34

4.1 Multivariable reductions of the dKP hierarchy . . . . . . . . . . . . 34

4.2 Multivariable reductions of the dBKP hierarchy . . . . . . . . . . . 36

4.3 Multivariable reductions of the dToda hierarchy . . . . . . . . . . . 38

4.4 Multivariable reductions of the dDKP hierarchy in the elliptic form . . . 39

4.4.1 The Gibbons-Tsarev equations and a system of hydrodynamic type 39

4.4 .2 Generalized hodograph method ... . . . . . . . . . 41

Acknowledgements

Appendix A: necessary functions and identities

References 


\section{Introduction}

Around the turn of the millennium it turned out that the hierarchies of integrable partial differential equations are related to the theory of univalent functions (i.e., to the Riemann mapping theorem) unexpectedly deeply. To wit, dispersionless limits of the KadomtsevPetviashvili (dKP) and the 2D Toda (dToda) hierarchies were shown to be closely related to conformal maps of domains in the complex plane.

This relation was developed in two seemingly different directions. One of them treats equations of the dToda hierarchy as governing equations for conformal maps of plane domains with smooth boundary as functions of their harmonic moments [1, 2]. Another one is related to conformal maps of domains with slits (slit domains). In the seminal papers [3, 4] reductions of the dKP hierarchy were studied and it was discovered that they are classified by solutions of a Löwner-type differential equation which characterizes one-parameter families of conformal mappings of domains with a growing slit and a fixed reference domain [5]. Later this important observation was extended to hierarchies of other types and other types of Löwner-like equations [6, 7, 8, 9, 10, 11].

The aim of this paper is to review reductions of the dispersionless hierarhies such as dKP, dToda as well as of the B- and D- versions of the dKP hierarchy (dBKP and dDKP) within a unified framework of Hirota's approach and to elucidate their deep relation with different types of the Löwner equation (respectively, chordal, radial, quadrant and elliptic). A conceptual understanding of mathematical origin of this relation is still missing.

The meaning of reduction is as follows. Infinite hierarchies of partial differential equations contain an infinite number of independent variables ("times") and an infinite number of dependent variables. The simplest possible reduction is a reduction to just one dependent variable which depends on all the times, and all other dependent variables become functions of it (a one-variable reduction). It appears that the one-variable reductions are described by solutions of a single Löwner equation with a driving function which characterizes the reduction. Geometrically, the driving function characterizes the shape of the slit and the single dependent variable is a parameter along the slit. One can also consider multi-variable $(N$-variable) reductions when there are $N$ dependent variables. In this case the reduction is described by a system of $N$ Löwner equations with $N$ driving functions and certain compatibility conditions (the Gibbons-Tsarev equations [3]) appear.

In section 2 we review the different types of Löwner equations from the point of view of complex analysis and conformal mappings. We would like to stress that the hierarchical sequence of Löwner equations

$$
\text { chordal } \longrightarrow \text { radial } \longrightarrow \text { elliptic }
$$

corresponds to the types of functions

$$
\text { rational } \longrightarrow \text { trigonometric } \longrightarrow \text { elliptic }
$$

and, on the side of dispersionless integrable hierarchies, to

$$
d K P \longrightarrow d T o d a \longrightarrow d D K P \text {. }
$$


In section 3 we consider dispersionless integrable hierarchies: dKP, dBKP, dToda and $\mathrm{dDKP}$ (the order corresponds to growing complexity). In each case we start from the generating equation for the dispersionless limit of the tau-function (coming from the Hirota bilinear equations) and in the cases of $\mathrm{dKP}, \mathrm{dBKP}$ and dToda prove that it is equivalent to the more familiar Lax formulation. (For the dDKP hierarchy in the elliptic form the Lax formulation is not known.) For each case, the one-variable reduction is considered and the corresponding Löwner equation (chordal, quadrant, radial and elliptic) is derived as the consistency condition of the reduction with the structure of the infinite hierarchy.

Section 4 is devoted to multi-variable reductions characterized by a system of Löwner equations. We derive the compatibility conditions for them which are the Gibbons-Tsarev equations. In the case of reduction, the infinite hierarchy is reduced to a finite system of differential equations of hydrodynamic type for a finite number of dependent variables. This system is implicitly solved by means of the generalized hodograph method developed by Tsarev [12].

\section{Löwner equations}

The conformal map of a domain with a slit of arbitrary shape to a reference domain (say, the upper half plane or the unit circle) satisfy certain differential equation as a function of a parameter characterizing the slit. Such differential equations are called equations of the Löwner type.

\subsection{Chordal Löwner equation}

The simplest equation of this type, now called chordal Löwner equation, was first obtained in [13]. Let $\Gamma$ be a smooth curve $\Gamma:[0,+\infty) \rightarrow \mathbb{H}$ in the upper half plane $\mathbb{H}$ starting from a point on the real axis, $\Gamma(0) \in \mathbb{R}$, and $\Gamma_{t}$ be its arc, $\Gamma_{t}:=\Gamma([0, t])$. We assume that, if the curve touches the real axis, it goes off and enters $\mathbb{H}$ immediately.

According to the Riemann mapping theorem, there exists a unique univalent conformal map $g(z, t)$ from $\mathbb{H} \backslash \Gamma_{t}$ to $\mathbb{H}$ normalized by the condition

$$
g(z, t)=z+u(t) z^{-1}+O\left(z^{-2}\right), \quad z \rightarrow \infty, \quad t>0 .
$$

The parameter $t$ along the curve is sometimes referred to as "time" and the coefficient $u(t)$ as the "capacity" of the domain $\mathbb{H} \backslash \Gamma_{t}$.

It can be shown (see, for example, [14]) that $u(t)$ is a continuous increasing function of $t$, which makes it possible to assume $u(t)=t$ by reparametrization. In this parametrization, $g(z, t)$ has the following form:

$$
g(z, t)=z+t z^{-1}+O\left(z^{-2}\right), \quad z \rightarrow \infty, \quad t>0 .
$$

It appears that there exists a continuous real-valued function $\xi(t)$ (called the driving function) such that $g(z, t)$ satisfies the differential equation

$$
\frac{\partial g(z, t)}{\partial t}=\frac{1}{g(z, t)-\xi(t)}, \quad g(z, 0)=z .
$$


任

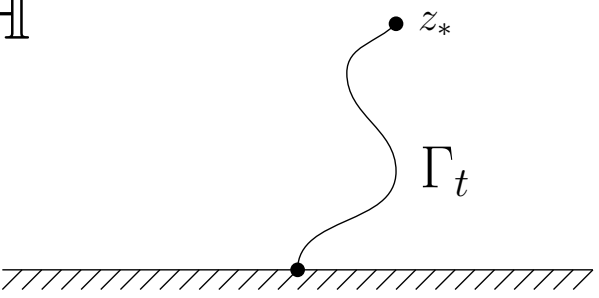

$\mathbb{H}$

$\stackrel{g(z, t)}{\longrightarrow}$

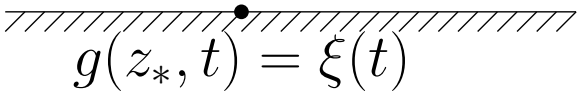

Figure 1:

This is the chordal Löwner equation. The point $\xi(t) \in \mathbb{R}$ is the image of the tip of the curve $\Gamma_{t}$. This is how the chordal Löwner equation appears in complex analysis. It was first proved in [13] and rediscovered in the context of integrable systems by Gibbons and Tsarev in [4. It became well-known when Schramm [15] discovered it independently and studied random curves in the upper half plane (in the celebrated SLE the driving function $\xi(t)$ is a Brownian motion).
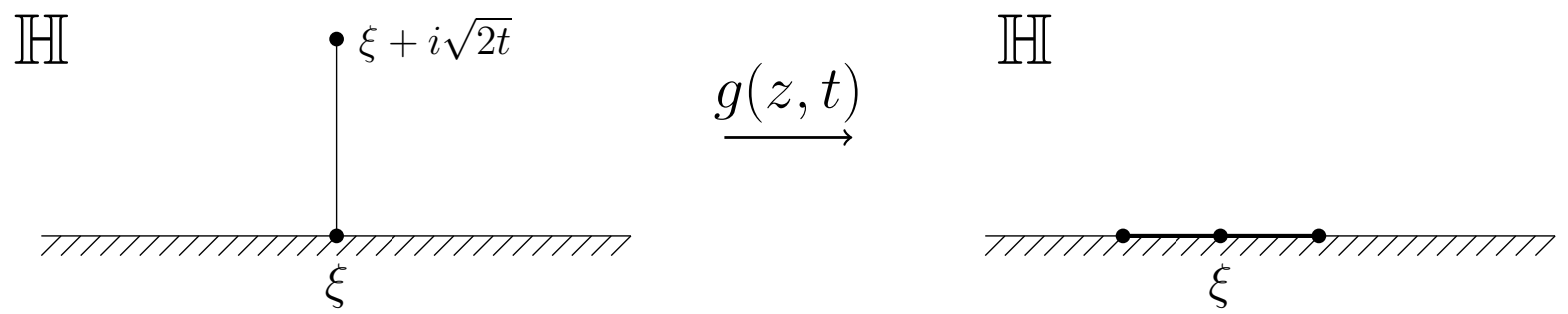

Figure 2:

For example, if $\Gamma_{t}=[\xi, \xi+i \sqrt{2 t}]$ is the straight segment from $\xi \in \mathbb{R}$ to the point $\xi+i \sqrt{2 t}$, then $\xi(t)=\xi=$ const and

$$
g(z, t)=\xi+\sqrt{(z-\xi)^{2}+2 t} .
$$

Note that if $t \rightarrow 0$ and $|z-\xi|$ is bounded from below, then

$$
g(z, t)=z+\frac{t}{z-\xi}+O\left(t^{2}\right)
$$

The origin of the equation (2.2) in a good situation (when, for example, the curve is smooth) is explained as follows [16]. (This explanation is by no means rigorous but simple and geometrically clear. For rigorous proofs we refer to, for example, [14], [17].)

Let the curve evolve for a "time" $t$ and then for a further short "time" $s, s \rightarrow 0$. The image of $\mathbb{H} \backslash \Gamma_{t+s}$ under $g(z, t+s)$ is $\mathbb{H}$ while the image of $\mathbb{H} \backslash \Gamma_{t+s}$ under $g(z, t)$ is $\mathbb{H}$ with a cut which is a short vertical segment starting from the point $\xi(t)$ on the real axis: The tip $z_{*}:=\Gamma(t)$ of the curve $\Gamma_{t}$ is mapped to $g\left(z_{*}, t\right)=\xi(t)$ by the map $g(z, t)$ (Figure 2). 

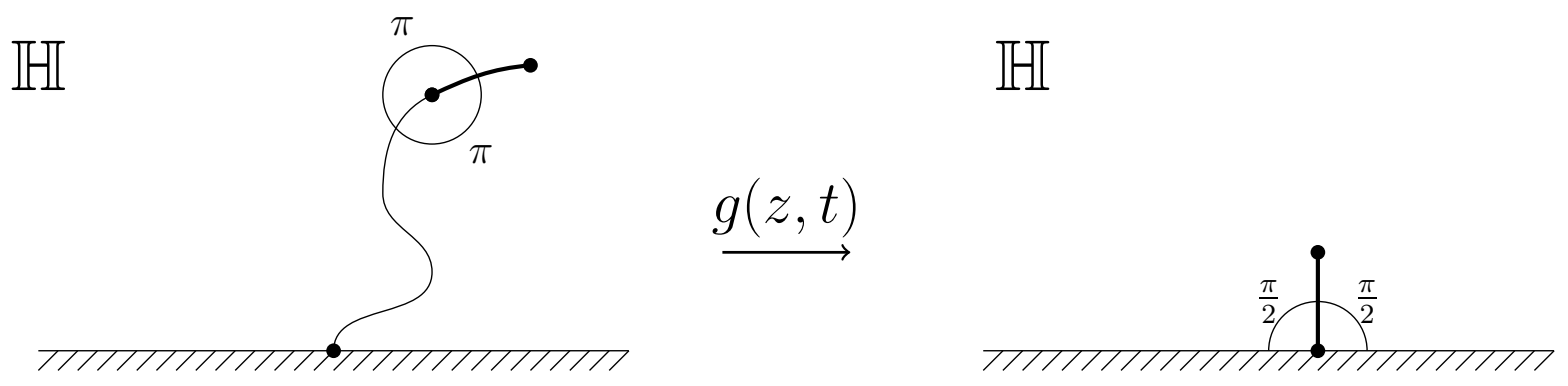

Figure 3:

The angle $2 \pi$ around $z_{*}$ shrinks to $\pi$ in the upper half plane around $\xi(t)$. From this argument we can infer that $g(z, t)$ behaves like (const.) $\times \sqrt{z-z_{*}}$ in the neighborhood of $z_{*}$. It follows that the angles $\pi$ on the both sides of the curve at the tip of $\Gamma_{t}$ (the left picture in Figure 3) shrinks to $\frac{\pi}{2}$ in the image (the right picture in Figure 3). Thus the complement of $\Gamma_{t}$ in $\Gamma_{t+s}$ (the bold curve in the left picture in Figure 3) is approximately mapped to a vertical segment (the bold segment in the right picture in Figure 3) by $g(z, t)$.

The map $g(z, t+s)$ is decomposed as $\left(g(z, t+s) \circ g(z, t)^{-1}\right) \circ g(z, t)$, where $g(z, t+$ $s) \circ g(z, t)^{-1}$ is the map from the domain in the right picture in Figure 3 to the upper half plane and approximated by the map in Figure 2, which justifies the following approximation:

$$
g(z, t+s) \circ g(z, t)^{-1}(w) \approx \xi(t)+\sqrt{(w-\xi(t))^{2}+2 c(t, s)}=w+\frac{c(t, s)}{w-\xi(t)}+O\left(w^{-2}\right),
$$

where $c(t, s)$ is determined by the length of the segment in the right picuture in Figure 3 . On the other hand, because of the normalization (2.1), we can expand $g(z, t+s) \circ g(z, t)^{-1}$ as follows:

$$
g(z, t+s) \circ g(z, t)^{-1}(w)=w+s w^{-1}+O\left(w^{-2}\right) .
$$

Comparing the coefficients, we obtain $c(t, s) \approx s$.

Therefore, we can write, using (2.3), (2.4),

$$
g(z, t+s) \approx \xi(t)+\sqrt{(g(z, t)-\xi(t))^{2}+2 s}=g(z, t)+\frac{s}{g(z, t)-\xi(t)}+O\left(s^{2}\right)
$$

which is equivalent to (2.2).

Another explanation (or the idea of the proof in [17], which follows the original idea by Kufarev et al., [13]; see also [18]) is as follows. The key ingredient is the Schwarz integral formula in complex analysis, a corollary of the Cauchy integral formula:

$$
f(z)=\frac{1}{\pi} \int_{\mathbb{R}} \frac{\operatorname{Im} f(\xi)}{\xi-z} d \xi
$$

where $f: \mathbb{H} \rightarrow \mathbb{C}$ is a holomorphic function on the upper half plane continuously extendable to $\overline{\mathbb{H}} \rightarrow \mathbb{C}$ and satisfies the following estimate:

$$
\lim _{R \rightarrow \infty} \max _{\varphi \in[0, \pi]}\left|f\left(R e^{i \varphi}\right)\right|=0
$$


In fact, applying the Cauchy integral formula to the semi-circle $[-R, R] \cup\left\{R e^{i \varphi} \mid \varphi \in\right.$ $[0, \pi]\}$, we have

$$
\begin{aligned}
f(z) & =\frac{1}{2 \pi i} \int_{-R}^{R} \frac{f(x)}{x-z} d x+\frac{1}{2 \pi} \int_{0}^{\pi} \frac{f\left(R e^{i \varphi}\right)}{R e^{i \varphi}-z} R e^{i \varphi} d \varphi, \\
0 & =\frac{1}{2 \pi i} \int_{-R}^{R} \frac{f(x)}{x-\bar{z}} d x+\frac{1}{2 \pi} \int_{0}^{\pi} \frac{f\left(R e^{i \varphi}\right)}{R e^{i \varphi}-\bar{z}} R e^{i \varphi} d \varphi .
\end{aligned}
$$

Taking the complex conjugate of the second equation and summing with the first, we obtain

$$
f(z)=\frac{1}{\pi} \int_{-R}^{R} \frac{\operatorname{Im} f(x)}{x-z} d x+\frac{1}{2 \pi} \int_{0}^{\pi} \frac{f\left(R e^{i \varphi}\right)}{R e^{i \varphi}-z} R e^{i \varphi} d \varphi+\frac{1}{2 \pi} \int_{0}^{\pi} \frac{\overline{f\left(R e^{i \varphi}\right)}}{R e^{-i \varphi}-z} R e^{-i \varphi} d \varphi .
$$

Because of the estimate (2.6), the last two terms converges to 0 when $R$ tends to infinity. Thus (2.5) is proved. (See also Proposition 2.2 of [17].)

Let us return to the chordal Löwner equation. For $0 \leq s \leq t$ we define a map $h(z ; s, t): \mathbb{H} \rightarrow \mathbb{H}$ by

$$
h(z ; s, t):=g\left(g^{-1}(z, t), s\right) .
$$

(See Figure 4.)

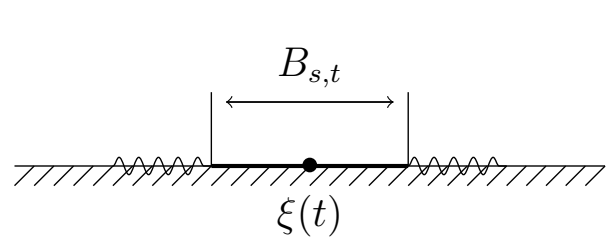

$$
\stackrel{h(\xi ; s, t)}{\longrightarrow}
$$
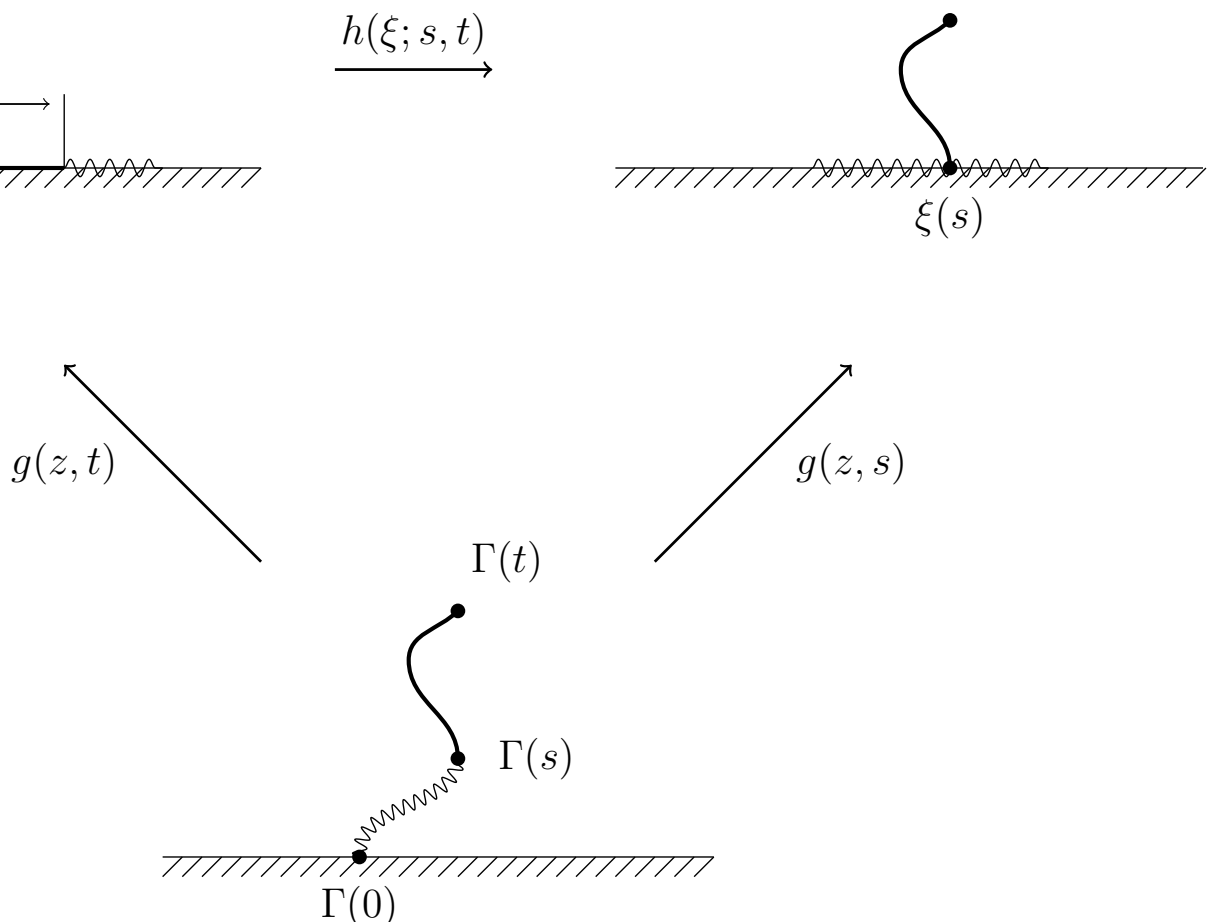

Figure 4:

If we apply (2.5) to $h(\xi ; s, t)-\xi$, we obtain

$$
h(\xi ; s, t)-\xi=\frac{1}{\pi} \int_{\mathbb{R}} \frac{\operatorname{Im}(h(x ; s, t)-x)}{x-\xi} d x .=\frac{1}{\pi} \int_{B_{s, t}} \frac{\operatorname{Im}(h(x ; s, t))}{x-\xi} d x .
$$


since the integration variable $x$ is real and the imaginary part of $h(z ; s, t)$ is non-zero only on the bold interval $B_{s, t}$ in Figure 4 . Setting $\xi \mapsto g(z, t)$, we have

$$
g(z ; s)-g(z, t)=\frac{1}{\pi} \int_{B_{s, t}} \frac{\operatorname{Im}(h(x ; s, t))}{x-g(z, t)} d x .
$$

On the other hand, setting $z=i y(y>0)$ in (2.8) and taking the limit $y \rightarrow \infty$, we have

$$
t-s=\frac{1}{\pi} \int_{B_{s, t}} \operatorname{Im}(h(x ; s, t)) d x
$$

since $h(\xi ; s, t)=\xi+(s-t) \xi^{-1}+O\left(\xi^{-2}\right)$.

The ratio of (2.9) and (2.10) gives

$$
\frac{g(z, s)-g(z, t)}{s-t}=\frac{\int_{B_{s, t}} \frac{\operatorname{Im} h(x ; s, t)}{x-g(z, t)} d x}{-\int_{B_{s, t}} \operatorname{Im} h(x ; s, t) d x}
$$

for $0 \leq s<t$

When $s$ approaches to $t$ from below, $s \nearrow t$, the left hand side of (2.11) converges to the left derivative of $g(z, t)$ with respect to $t$. The segment $B_{s, t}$ shrinks to $\xi(t)$ in this

limit, which means that the right hand side of (2.11) converges to $\frac{1}{g(z, t)-\xi(t)}$. Thus we have an equation for $h(\xi ; s, t)$ :

$$
\frac{\partial}{\partial t} g(z, t)=\frac{1}{g(z, t)-\xi(t)}
$$

where the differentiation is understood as the left derivative. We can also prove the same differential equation with the right derivative, and therefore the above equation holds as a usual differential equation. namely, (2.2).

Below in section 3.1 .2 we will derive the chordal Löwner equation in an absolutely different context of integrable hierarchies of nonlinear partial differential equations in the dispersionless limit as the consistency condition of the infinite dKP hierarchy with one-variable reduction.

\subsection{Quadrant Löwner equation}

Let us take the quadrant $Q=\{z \mid \operatorname{Re} z>0, \operatorname{Im} z>0\}$ as the reference domain for the chordal Löwner equation instead of $\mathbb{H}$. Namely, we identify $\mathbb{H}$ with $Q$ by a mapping of the form $h_{a}: Q \ni z \mapsto \tilde{z}=z^{2}-a(t) \in \mathbb{H}(a(t) \in \mathbb{R})$ and rewrite the equation (2.2).

If the function $g(z, t)$ of the form

$$
g(z, t)=z+\sum_{n=1}^{\infty} v_{n}(t) z^{1-2 n}
$$

satisfies the equation

$$
\frac{\partial g}{\partial t}=-\frac{g}{g^{2}-\xi^{2}} \frac{d u}{d t}
$$


$\left(u=-v_{1}\right)$, then $\tilde{g}(\tilde{z}, t):=g(\sqrt{\tilde{z}}, t)^{2}-2 v_{1}(t)$ satisfies the hydrodynamic normalization condition

$$
\tilde{g}(\tilde{z}, t)=\tilde{z}+\tilde{u}(t) \tilde{z}^{-1}+O\left(\tilde{z}^{-2}\right)
$$

$\left(\tilde{u}=2 v_{2}+v_{1}^{2}\right)$ and the chordal Löwner equation:

$$
\frac{\partial \tilde{g}}{\partial t}=\frac{1}{\tilde{g}-\tilde{\xi}} \frac{d \tilde{u}}{d t} .
$$

Here the driving function $\tilde{\xi}=\tilde{\xi}(t)$ is

$$
\tilde{\xi}(t)=\xi(t)^{2}-2 v_{1}(t)
$$

Moreover, $\tilde{u}(t)$ satisfies

$$
\frac{d \tilde{u}}{d t}=-2 \xi^{2} \frac{d u}{d t}
$$

Conversely, let $\tilde{g}(\tilde{z}, t)=\tilde{z}+\tilde{u}(t) \tilde{z}^{-1}+\ldots$ be a solution of the chordal Löwner equation (2.15) with the driving function $\tilde{\xi}(t)$. Let $\xi(t)$ be a solution of the following ordinary differential equation 1 :

$$
\frac{d}{d t} \xi^{4}-2 \frac{d \tilde{\xi}}{d t} \xi^{2}=2 \frac{d \tilde{u}}{d t}
$$

If we define $u(t)$ by

$$
u(t)=\frac{1}{2}\left(\tilde{\xi}(t)-\xi(t)^{2}\right),
$$

then $g(z, t):=\sqrt{\tilde{g}\left(z^{2}, t\right)-2 u(t)}$ is of the form (2.12) and satisfies equation (2.13). (Exactly speaking, we can choose a branch of the square root so that $g(z ; t)$ is of the form (2.12) and satisfies (2.13).)

The proof is a straightforward computation (see [10], Proposition 5.1).

We see that a one-parameter family of conformal mappings from $Q \backslash$ (slit) to $Q$ with the normalization (2.12) (the upper half of Figure 5) satisfies the equation (2.13), which we call the quadrant Löwner equation. Note that such a conformal map should be an odd function because of the Schwarz reflection principle and therefore has the form (2.12) up to normalization.

The quadrant Löwner equation adds nothing essential to the Löwner theory, but is related to the dispersionless BKP hierarchy, as we shall see later. In fact, the quadrant Löwner equation was intorduced in this context in [10]. (For another application see [20].)

\subsection{Radial Löwner equation}

The radial version of the Löwner equation differs from the chordal one by a different normalization. Let $\Gamma_{t}$ be an arc of a smooth curve in the exterior of the unit disk $\mathbb{U}^{c}$

\footnotetext{
${ }^{1}$ The ordinary differential equation (2.18) for $\xi^{2}$ is a special case of Chini's equation (C.I.55 of [19], p.303; $\left.x \mapsto t, y \mapsto \xi(t)^{2}, n=-1, f(x) \mapsto d \tilde{u} / d t, g(x) \mapsto 0, h(x) \mapsto d \tilde{\xi} / d t\right)$. It can be solved explicitly only in special cases.
} 

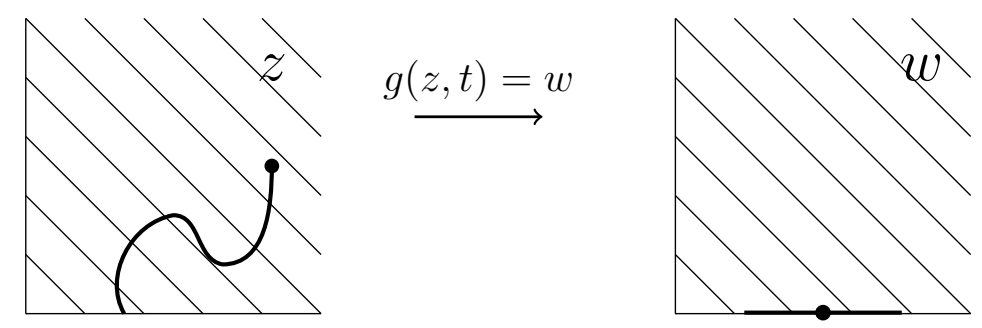

$$
{ }^{\xi(t)} w=\sqrt{\tilde{w}-2 u(t)}
$$$$
\downarrow \tilde{z}=z^{2}
$$
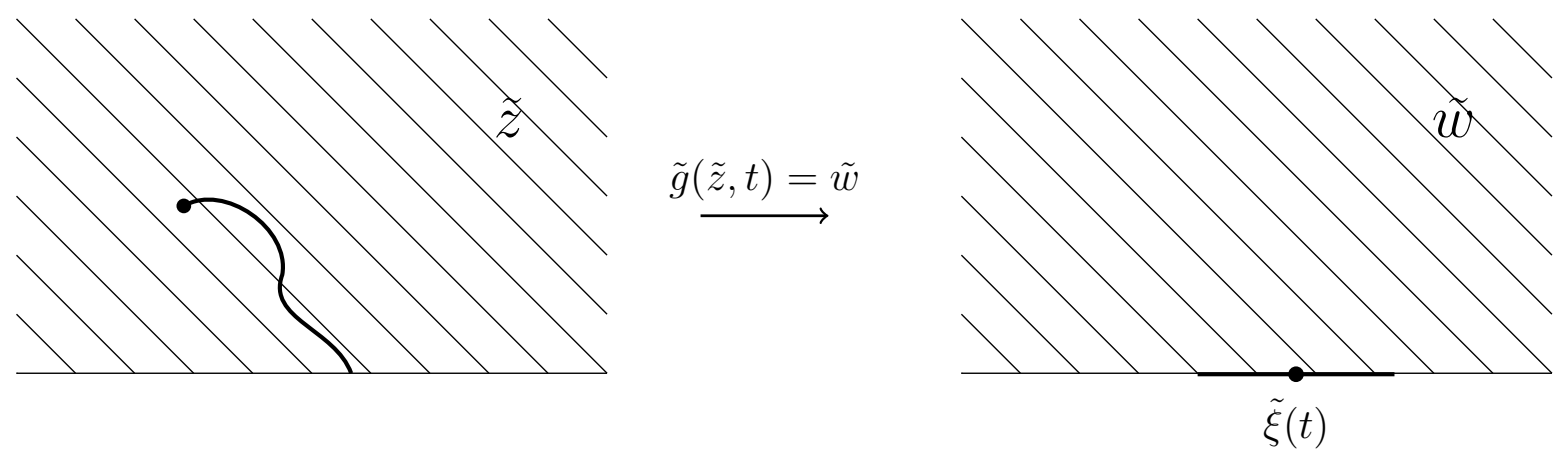

Figure 5:

starting from a point on the unit circle $\mathbb{S}$, and let $g(z, t)$ be the univalent conformal map from $\mathbb{U}^{c} \backslash \Gamma_{t}$ to $\mathbb{U}^{c}$ normalized by the condition

$$
g(z, t)=e^{-\phi(t)} z+O(1), \quad z \rightarrow \infty, \quad t \in \mathbb{R} .
$$

According to the Riemann mapping theorem, such a map exists and is unique. The quantity $r=e^{\phi(t)}$ is called the conformal radius of the domain $\mathbb{U}^{c} \backslash \Gamma_{t}$. As before we reparametrize the curve so that $\phi(t)=t$ :

$$
g(z, t)=e^{-t} z+O(1), \quad z \rightarrow \infty, \quad t \in \mathbb{R} .
$$

Note that a fixed interior point $z=\infty$ of $\mathbb{U}^{c}$ is mapped to a fixed inner point $\infty$, while in the chordal case a fixed boundary point $z=\infty$ is mapped to a fixed boundary point $\infty$. This difference in normalization is the essential difference between the two types of the Löwner equation.

It appears that there exists a continuous real-valued function $\xi(t)$ (called the driving function) such that $g(z, t)$ satisfies the differential equation

$$
\frac{\partial g(z, t)}{\partial t}=-g(z, t) \frac{g(z, t)+\eta(t)}{g(z, t)-\eta(t)}, \quad g(z, 0)=z, \quad \eta(t)=e^{i \xi(t)}
$$

It is now called the radial Löwner equation to distinguish it from the chordal case. This equation was found by Löwner in 1923 [5]. It is a powerful tool in geometric function theory, for example, to evaluate coefficients of univalent functions. One of the examples, 
to which the radial Löwner equation was successfully applied, is the famous Bieberbach conjecture.

For example, if $\Gamma_{t}=[\eta, \eta \rho]$ is the straight segment from $\eta \in \mathbb{S}$ to the point $\eta \rho$ (orthogonal to the unit circle), where

$$
\rho=2 e^{t}-1+2 \sqrt{e^{2 t}-e^{t}} \in \mathbb{R}
$$

then $\eta(t)=\eta=$ const and

$$
g(z, t)=-\eta+\frac{1}{2} e^{-t}(z+\eta)\left(1+\frac{\eta}{z}+\frac{1}{z} \sqrt{z^{2}+2 \eta z\left(1-2 e^{t}\right)+\eta^{2}}\right) .
$$

Note that $g(\eta \rho, t)=\eta$ and that if $t \rightarrow 0$ and $|z-\eta|$ is bounded from below, then

$$
g(z, t)=z-z \frac{z+\eta}{z-\eta} t+O\left(t^{2}\right)
$$

We can explain (2.20) in the same way as in the chordal case.
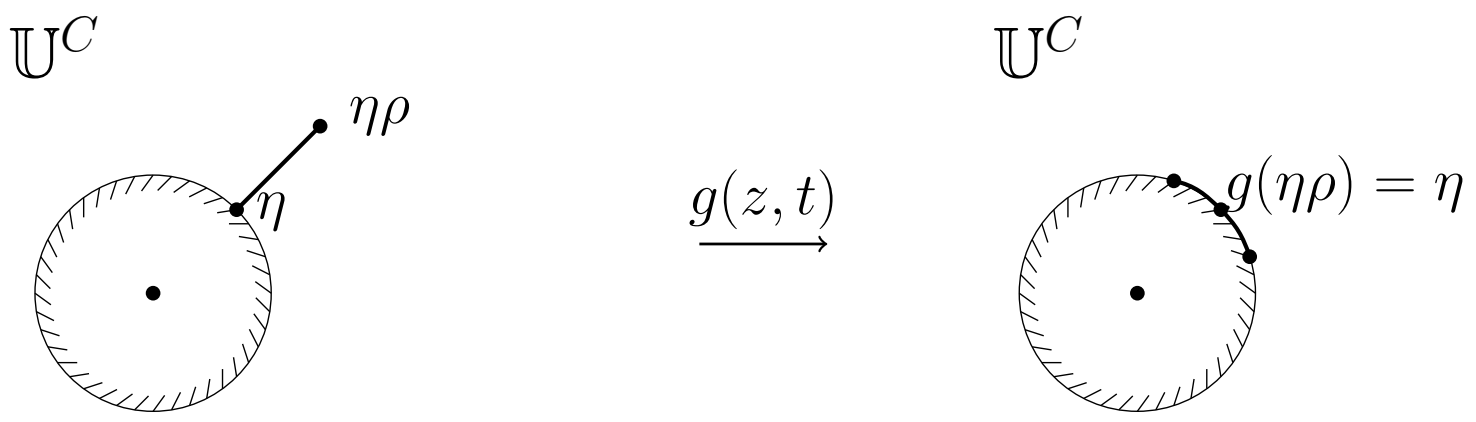

Figure 6:

Let the curve evolve for a "time" $t$ and then for a further short "time" $s, s \rightarrow 0$. The image of $\mathbb{U}^{c} \backslash \Gamma_{t+s}$ under $g(z, t)$ is $\mathbb{U}^{c}$ with a cut which is a short segment orthogonal to the unit circle starting from the point $\eta(t)$ on $\mathbb{S}$. Therefore, we can write, using (2.21), (2.22),

$$
g(z, t+s) \approx g(z, t)-g(z, t) \frac{g(z, t)+\eta(t)}{g(z, t)-\eta(t)} s+O\left(s^{2}\right)
$$

which is equivalent to (2.20).

In another explanation (the idea of the proof in, e.g., [21], [22], [23] §34) the complex Poincaré integral formula

$$
f(z)=\frac{1}{2 \pi} \int_{0}^{2 \pi} \operatorname{Re} f\left(e^{i \theta}\right) \frac{e^{i \theta}+z}{e^{i \theta}-z} d \theta+i \operatorname{Im} f(0)
$$

for a holomorphic function $f(z)$ on the unit disk $\mathbb{U}$ plays the role of the Schwarz integral formula (2.5) in the chordal case. 
Let us consider a family of conformal mappings $f(z, t)$ from $\mathbb{U} \backslash \Gamma((0, t])$ to $\mathbb{U}$. Here $\Gamma:[0,+\infty) \rightarrow \overline{\mathbb{U}}$ is a Jordan curve with the condition $|\Gamma(0)|=1$ and $f(z, t)$ is normalized by $f(z, t)=e^{t} z+O\left(z^{2}\right)$. We derive the differential equation

$$
\frac{\partial f(z, t)}{\partial t}=f(z, t) \frac{\lambda(t)+f(z, t)}{\lambda(t)-f(z, t)}
$$

from which equation (2.20) follows immediately $\left(g(z, t)=1 / f\left(z^{-1}, t\right), \eta(t)=1 / \lambda(t)\right)$.

As in the chordal case (cf. (2.7) $)$, we define a map $h(z ; s, t)$ for $z \in \mathbb{U}(0<s<t)$ by

$$
h(z ; s, t):=f\left(f^{-1}(z, t), s\right)=e^{s-t} z+O\left(z^{2}\right) .
$$

In Figure 7 the image of the tip of the curve by $f(z, t)$ is denoted by $\lambda(t): \lambda(t)=f(\Gamma(t), t)$.

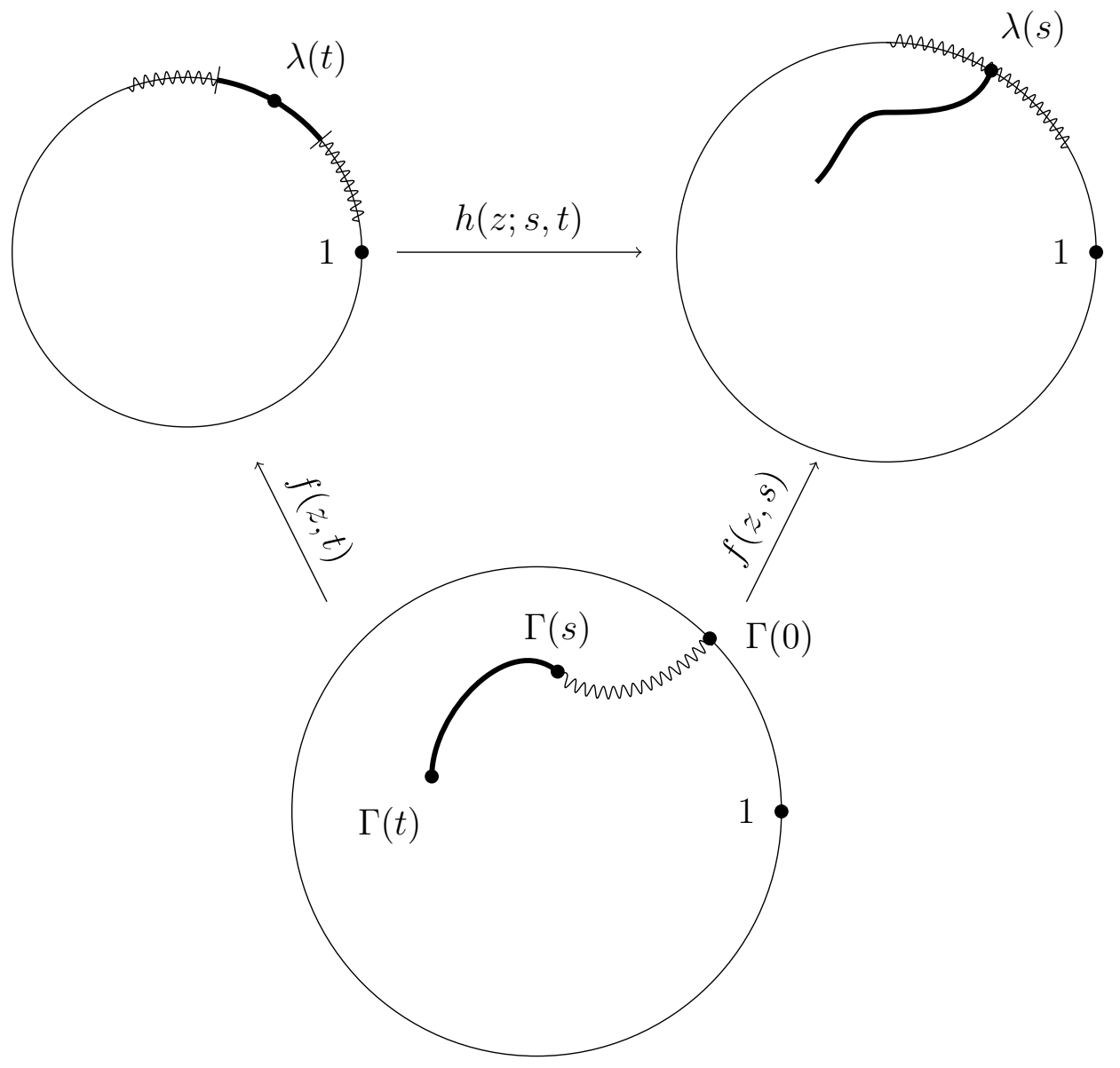

Figure 7:

Applying (2.23) to the function $\log (h(z ; s, t) / z)$, we have

$$
\log \frac{h(z ; s, t)}{z}=\frac{1}{2 \pi} \int_{0}^{2 \pi} \log \left|h\left(e^{i \theta} ; s, t\right)\right| \frac{e^{i \theta}+z}{e^{i \theta}-z} d \theta,
$$

and, putting $z \mapsto f(z, t)$,

$$
\log \frac{f(z, s)}{f(z, t)}=\frac{1}{2 \pi i} \int_{0}^{2 \pi} \log \left|h\left(e^{i \theta} ; s, t\right)\right| \frac{e^{i \theta}+f(z, t)}{e^{i \theta}-f(z, t)} d \theta,
$$


which corresponds to (2.9) in the chordal case.

On the other hand, putting $z=0$ in (2.26), we have

$$
s-t=\frac{1}{2 \pi} \int_{0}^{2 \pi} \log \left|h\left(e^{i \theta} ; s, t\right)\right| d \theta,
$$

which substitutes (2.10) in the chordal case.

Note that $\log \left|h\left(e^{i \theta} ; s, t\right)\right|=0$ if $e^{i \theta}$ is mapped to the boundary of $\mathbb{U}$ by $h(z ; s, t)$, namely, if it does not lie on the bold arc of the left upper circle in Figure 2.3. Since the bold arc shrinks to $\lambda(t)$, when $s \nearrow t$, the ratio of (2.27) and (2.28) converges to

$$
\frac{\partial \log f(z, t)}{\partial t}=\frac{\lambda(t)+f(z, t)}{\lambda(t)-f(z, t)},
$$

where the differentiation in the left hand side is understood as the left derivative. We can also prove the same differential equation with the right derivative, and therefore the above equation holds as a usual differential equation. Thus we have obtained (2.24).

Below in section 3.3 .2 we will derive the radial Löwner equation in the context of integrable hierarchies as the consistency condition of the infinite dToda hierarchy with one-variable reduction.

\subsection{Komatu-Löwner equation}

The next example of the Löwner-type equations is an equation for the doubly connected domain found by Komatu ([24], [25] §84). (See also [26, 27, 28, 29, 30, 31]2.)

Let $Q$ be a positive real number, $0<Q<1, \mathbb{A}_{Q}$ be an annulus, $\left\{z|1<| z \mid<Q^{-1}\right\}$ and $\Gamma$ be a Jordan curve in the closure of $\mathbb{A}_{Q}, \Gamma:[0,+\infty) \rightarrow \mathbb{A}_{Q}$. We assume that $\Gamma$ starts from the outer boundary of $\mathbb{A}_{Q},|\Gamma(0)|=Q^{-1}$, and the other part lies completely in $\mathbb{A}_{Q}, \Gamma((0,+\infty)) \subset \mathbb{A}_{Q}$. Then, as is known in the geometric function theory, we can reparametrize the curve, so that there exists a unique conformal map $g(z, q)$ for each $q \in[0,+\infty)$ which maps $\mathbb{A}_{Q} \backslash \Gamma((0, q])$ onto $\mathbb{A}_{q}=\left\{w|1<| w \mid<q^{-1}\right\}$ with the normalization condition,

$$
g(1, q)=1
$$

Let $\Lambda(q)$ be the image of $\Gamma(q)$ by $g(z, q)$,

$$
\Lambda(q):=g(\Gamma(q), q)
$$

Then $g(z, q)$ satisfies the following Komatu-Löwner equation $3^{3}$.

$$
\begin{aligned}
\frac{\partial \log g(z, q)}{\partial \log q} & =\mathcal{K}_{q}(g(z, q), \Lambda(q))-\mathcal{K}_{q}(1, \Lambda(q)) \\
& =-\frac{1}{\pi i}\left(\zeta_{1}\left(\frac{\log g(z, q)-\log \Lambda(q)}{2 \pi i}, \frac{\log q}{\pi i}\right)+\zeta_{1}\left(\frac{\log \Lambda(q)}{2 \pi i}, \frac{\log q}{\pi i}\right)\right)
\end{aligned}
$$

\footnotetext{
${ }^{2}$ We partly follow the normalizations of maps and annuli in [26] and partly those in [31, so that the resulting differential equation has the same form as the reduction of the dispersionless DKP hierarchy in $\$ 3.4$.

${ }^{3}$ Sometimes it is called the Goluzin-Komatu equation (e.g., in [28, [32, [33]).
} 

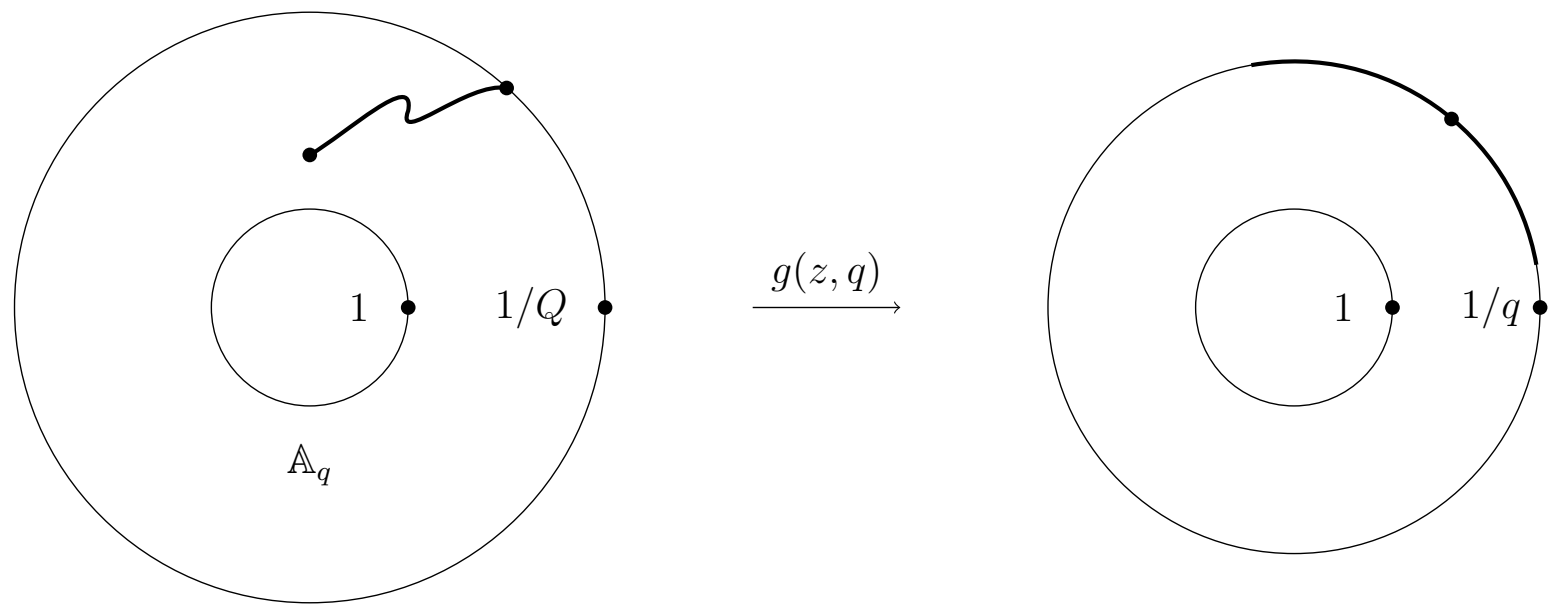

Figure 8:

The functions $\mathcal{K}_{q}(z, \zeta)$ (Villat's kernel) and $\zeta_{1}(u, \tau)$ are defined by (A7) and (A5) respectively. The expression with $\zeta_{1}$ obtained from the expression with $\mathcal{K}_{q}$ by the relations (A6) and $\zeta_{1}(-u)=-\zeta_{1}(u)$.

The idea of the proof is essentially the same as in the chordal Löwner case. The substitute of the Schwarz integral formula (2.5) is Villat's formula: Let $f: \overline{\mathbb{A}}_{q} \rightarrow \mathbb{C}$ be a continuous function, which is holomorphic on $\mathbb{A}_{q}$. Then $f(z)$ is expressed as follows:

$$
\begin{aligned}
f(z)= & \frac{1}{2 \pi} \int_{0}^{2 \pi} \operatorname{Re} f\left(q^{-1} e^{i \theta}\right) \mathcal{K}_{q}\left(z, q^{-1} e^{i \theta}\right) d \theta-\frac{1}{2 \pi} \int_{0}^{2 \pi} \operatorname{Re} f\left(e^{i \theta}\right) \mathcal{K}_{q}\left(z, e^{i \theta}\right) d \theta \\
& -\frac{1}{2 \pi} \int_{0}^{2 \pi} \operatorname{Re} f\left(e^{i \theta}\right) d \theta+\frac{1}{2 \pi} \int_{0}^{2 \pi} \operatorname{Im} f\left(e^{i \theta}\right) d \theta .
\end{aligned}
$$

Furthermore,

$$
\int_{0}^{2 \pi} \operatorname{Re} f\left(e^{i \theta}\right) d \theta=\int_{0}^{2 \pi} \operatorname{Re} f\left(e^{i \theta}\right) d \theta .
$$

Assume that $\operatorname{Re} f(z)=: A \in \mathbb{R}$ is constant on the inner boundary $\partial_{\text {inner }} \mathbb{A}_{q}=\{z|| z \mid=1\}$ of $\mathbb{A}_{q}$. Villat's formula gives the following expression of $f(z)$ :

$$
f(z)=\frac{1}{2 \pi} \int_{0}^{2 \pi} \operatorname{Re} f\left(e^{i \theta}\right) \mathcal{K}_{q}\left(z, e^{i \theta}\right) d \theta+i c,
$$

where $c$ is a real constant. Moreover the constant $A$ is expressed by the following integral:

$$
A=\frac{1}{2 \pi} \int_{0}^{2 \pi} \operatorname{Re} f\left(q^{-1} e^{i \theta}\right) d \theta
$$

As in the chordal case (cf. (2.7) $)$, we define a map $h\left(\xi ; q^{*}, q\right)$ for $\xi \in \mathbb{A}_{q}\left(0<q^{*}<q<\right.$ 1) by

$$
h\left(\xi ; q^{*}, q\right):=g\left(g^{-1}(\xi, q), q^{*}\right) .
$$

(See Figure 9.)

Applying (2.35) to the function

$$
\Phi(\xi):=\log \frac{h\left(\xi ; q^{*}, q\right)}{\xi},
$$




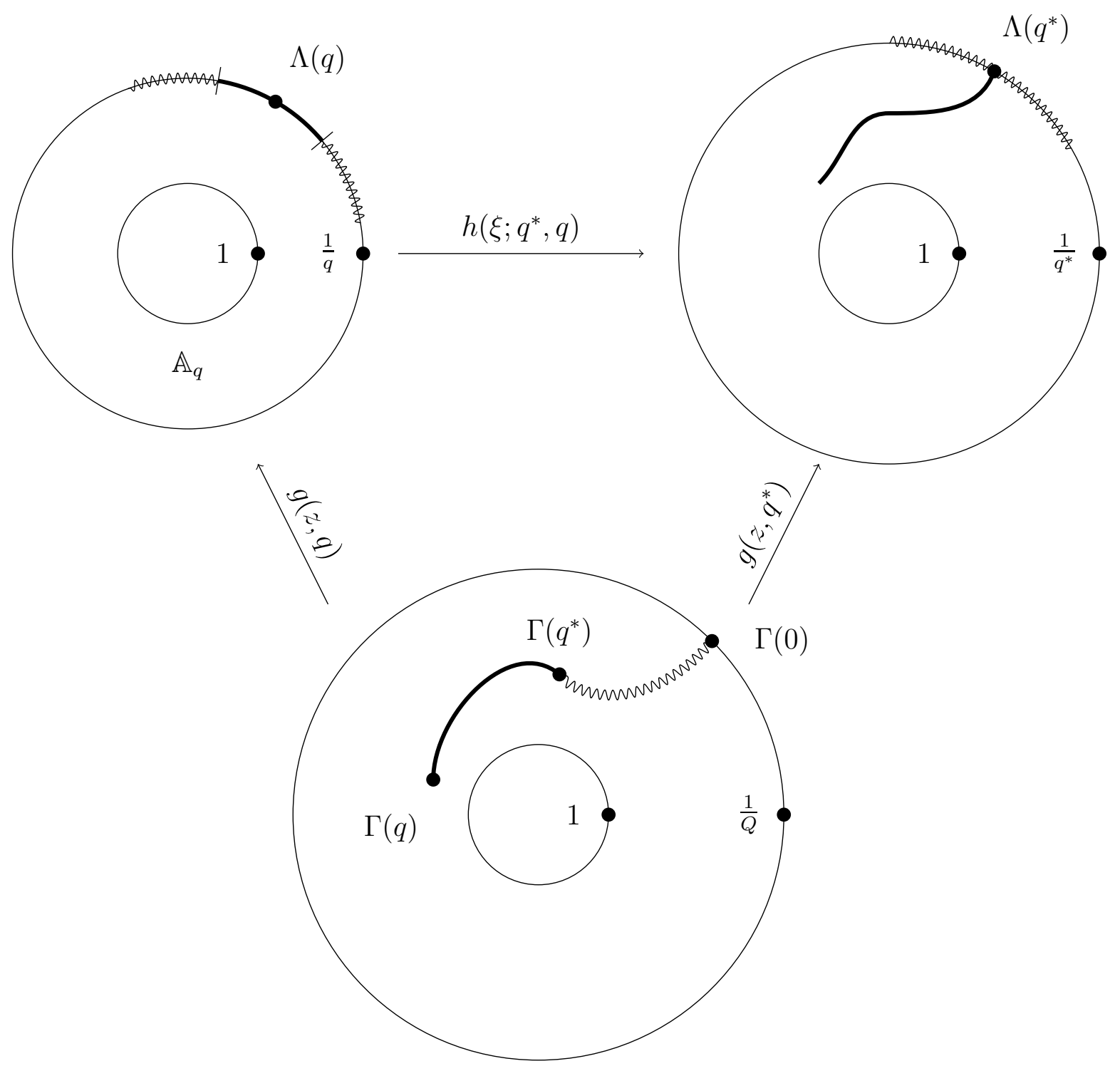

Figure 9:

we have

$$
\log \frac{h\left(\xi ; q^{*}, q\right)}{\xi}=\frac{1}{2 \pi} \int_{0}^{2 \pi} \log \left|q h\left(q^{-1} e^{i \theta} ; q^{*}, q\right)\right| \mathcal{K}_{q}\left(\xi, q^{-1} e^{i \theta}\right) d \theta+i c
$$

and, putting $\xi \mapsto g(z, q)$,

$$
\log \frac{g\left(z, q^{*}\right)}{g(z, q)}=\frac{1}{2 \pi} \int_{0}^{2 \pi} \log \left|q h\left(q^{-1} e^{i \theta} ; q^{*}, q\right)\right| \mathcal{K}_{q}\left(g(z, q), q^{-1} e^{i \theta}\right) d \theta+i c .
$$

It is easy to see that

$$
1=\frac{1}{2 \pi} \int_{0}^{2 \pi} \mathcal{K}_{q}\left(w, q^{-1} e^{i \theta}\right) d \theta
$$

for any $w \in \mathbb{A}_{q}$ by a simple residue computation. Multipling $\log \left(q^{*} / q\right)$ to this equation and adding it to (2.39), we obtain

$$
\log \frac{g\left(z, q^{*}\right)}{g(z, q)}+\log \frac{q^{*}}{q}=\frac{1}{2 \pi} \int_{0}^{2 \pi} \log \left|q^{*} h\left(q^{-1} e^{i \theta} ; q^{*}, q\right)\right| \mathcal{K}_{q}\left(g(z, q), q^{-1} e^{i \theta}\right) d \theta+i c .
$$


This implies

$$
c=-\frac{1}{2 \pi} \int_{0}^{2 \pi} \log \left|q^{*} h\left(q^{-1} e^{i \theta} ; q^{*}, q\right)\right| \operatorname{Im} \mathcal{K}_{q}\left(1, q^{-1} e^{i \theta}\right) d \theta .
$$

by the normalization $g(1, q)=g\left(1, q^{*}\right)=1$ and $\log \left(q^{*} / q\right) \in \mathbb{R}$. Therefore (2.40) is rewritten as

$$
\begin{aligned}
& \log \frac{g\left(z, q^{*}\right)}{g(z, q)}+\log \frac{q^{*}}{q} \\
& \quad=\frac{1}{2 \pi} \int_{0}^{2 \pi} \log \left|q^{*} h\left(q^{-1} e^{i \theta} ; q^{*}, q\right)\right|\left(\mathcal{K}_{q}\left(g(z, q), q^{-1} e^{i \theta}\right)-i \operatorname{Im} \mathcal{K}_{q}\left(1, q^{-1} e^{i \theta}\right)\right) d \theta
\end{aligned}
$$

which corresponds to (2.9) in the chordal case.

On the other hand, applying (2.36) to the function $\Phi(\xi)$, we have

$$
0=\frac{1}{2 \pi} \int_{0}^{2 \pi} \log \left|\frac{h\left(q^{-1} e^{i \theta} ; q^{*}, q\right)}{q^{-1} e^{i \theta}}\right| d \theta=\log q+\frac{1}{2 \pi} \int_{0}^{2 \pi} \log \left|h\left(q^{-1} e^{i \theta} ; q^{*}, q\right)\right| d \theta .
$$

Adding $\log q^{*}$ to the above equation, we obtain

$$
\log \frac{q^{*}}{q}=\frac{1}{2 \pi} \int_{0}^{2 \pi} \log \left|q^{*} h\left(q^{-1} e^{i \theta} ; q^{*}, q\right)\right| d \theta
$$

which substitutes (2.10) in the chordal case.

Note that $\log \left|q^{*} h\left(q^{-1} e^{i \theta} ; q^{*}, q\right)\right|=0$ if $q^{-1} e^{i \theta}$ is mapped to the boundary of $\mathbb{A}_{q^{*}}$ by $h\left(\xi ; q^{*}, q\right)$, namely, if it does not lie on the bold arc of the left upper annulus in Figure 9 . Since the bold arc shrinks to $\Lambda(q)$, when $q^{*} \nearrow q$, the ratio of (2.42) and (2.44) converges to

$$
\frac{\partial \log g(z, q)}{\partial \log q}+1=\mathcal{K}_{q}(g(z, q), \Lambda(q))-i \operatorname{Im} \mathcal{K}_{q}(1, \Lambda(q))
$$

where the differentiation in the left hand side is understood as the left derivative. We can also prove the same differential equation with the right derivative, and therefore the above equation holds as a usual differential equation.

The equation (2.45) apparently differs from the Komatu-Löwner equation (2.32), but we can rewrite (2.45) to (2.32), following the observation in [26]. Putting $z=1$ in (2.45), we have

$$
1=\mathcal{K}_{q}(1, \Lambda(q))-i \operatorname{Im} \mathcal{K}_{q}(1, \Lambda(q))
$$

which means $\operatorname{Re} \mathcal{K}_{q}(1, \Lambda(q))=1$. Hence, "1" in the left hand side of (2.45) can be moved to the right hand side as " $\operatorname{Re} \mathcal{K}_{q}(1, \Lambda(q))$ ". Thus we obtain the Komatu-Löwner equation,

$$
\frac{\partial \log g(z, q)}{\partial \log q}=\mathcal{K}_{q}(g(z, q), \Lambda(q))-\mathcal{K}_{q}(1, \Lambda(q)) .
$$




\section{One-variable reductions of integrable hierarchies}

\subsection{One-variable reductions of the dKP hierarchy}

\subsubsection{The dKP hierarchy}

Let $\mathbf{t}=\left\{t_{1}, t_{2}, t_{3}, \ldots\right\}$ be an infinite set of independent variables ("times") which are supposed to be real 4 . It is convenient to introduce the differential operator

$$
D(z)=\sum_{k \geq 1} \frac{z^{-k}}{k} \partial_{t_{k}} .
$$

The dKP hierarchy in the Hirota form can be written as the equation

$$
e^{D\left(z_{1}\right) D\left(z_{2}\right) F}=1-\frac{\partial_{t_{1}} D\left(z_{1}\right) F-\partial_{t_{1}} D\left(z_{2}\right) F}{z_{1}-z_{2}}
$$

for the real-valued function $F=F(\mathbf{t})$ which should hold for any $z_{1}, z_{2}$ [18, 34, 35]. The differential equations of the hierarchy are obtained by expanding this equation in (inverse) powers of $z_{1}, z_{2}$. It is convenient to introduce the function

$$
p(z)=z-\partial_{t_{1}} D\left(z_{1}\right) F,
$$

the equation (3.2) acquires the form

$$
e^{D\left(z_{1}\right) D\left(z_{2}\right) F}=\frac{p\left(z_{1}\right)-p\left(z_{2}\right)}{z_{1}-z_{2}} .
$$

The function $p(z)$ clearly depends also on all the times: $p(z)=p(z ; \mathbf{t})$. One can expand $p(z)$ in a series of the form

$$
p(z)=z-\frac{u}{z}+\sum_{k \geq 2} \frac{u_{k}}{z^{k}},
$$

where

$$
u=u(\mathbf{t})=\partial_{t_{1}}^{2} F .
$$

The coefficients $u_{k}$ are dependent variables. They are real-valued functions of the times.

If the series $p(z)$ converges in some neighborhood of infinity and defined a function $p(z)$, it can be given a geometrical meaning as conformal map from the upper half plane with a "fat slit" to the upper half plane [36]. The times $t_{k}$ are harmonic moments of the (exterior of) the domain. The coefficient $u$ in the expansion (3.5) is called (logarithmic) capacity of the domain.

Let us take logarithms of both sides of (3.4),

$$
D\left(z_{1}\right) D\left(z_{2}\right) F=\log \frac{p\left(z_{1}\right)-p\left(z_{2}\right)}{z_{1}-z_{2}},
$$

and differentiate with respect to $t_{1}$. Taking into account (3.3), we get:

$$
D\left(z_{2}\right) p\left(z_{1}\right)=-\frac{\partial_{t_{1}} p\left(z_{1}\right)-\partial_{t_{1}} p\left(z_{2}\right)}{p\left(z_{1}\right)-p\left(z_{2}\right)},
$$

\footnotetext{
${ }^{4}$ In algebraic theory of the dKP hierarchy (see e.g. 34]) the variables are not necessarily real.
} 
from which it is seen that

$$
D\left(z_{2}\right) p\left(z_{1}\right)=D\left(z_{1}\right) p\left(z_{2}\right) .
$$

Tending $z_{2} \rightarrow \infty$, we get, using (3.5):

$$
\partial_{t_{1}} p(z)=-D(z) u \text {. }
$$

In what follows we assume that the series $p(z)$ converges in some neighborhood of infinity and defines a meromorphic function (conformal map) $p(z)$. Equation (3.7) acquires a more familiar form in terms of the inverse function to the $p(z)$, which we denote $z(p)$. It maps the upper half plane to the domain which is the complement in the upper half plane to a "fat slit". Similarly to (3.5), one can expand $z(p)$ into a series of the form

$$
z(p)=p+\frac{u}{p}+\sum_{k \geq 2} v_{k} p^{-k}
$$

In order to transform equation (3.7) to a more suggestive form, we introduce the polynomials $B_{k}(p)$ by the expansion

$$
-\log \frac{p(z)-p}{z}=\sum_{k \geq 1} \frac{z^{-k}}{k} B_{k}(p)
$$

They are called Faber polynomials [21]. For example, $B_{1}(p)=p$. It is easy to see that

$$
B_{k}(p)=\left(z^{k}(p)\right)_{\geq 0}
$$

where $(\ldots)_{\geq 0}$ is the polynomial part of the Laurent series in $p$ (containing only nonnegative powers of the variable). Indeed, let $z_{1}$ be the pre-image of the point $p$ under the map $p(z)$, then we can write

$$
\begin{gathered}
\log \left(p(z)-p\left(z_{1}\right)\right)=\log \frac{p(z)-p\left(z_{1}\right)}{z-z_{1}}+\log \left(z-z_{1}\right) \\
=\log \frac{p(z)-p\left(z_{1}\right)}{z-z_{1}}+\log z-\sum_{k \geq 1} \frac{z^{-k}}{k} z_{1}^{k} .
\end{gathered}
$$

Using the fact that $\left(\log \frac{p(z)-p\left(z_{1}\right)}{z-z_{1}}\right)_{\geq 0}=0$ and comparing with (3.11), we get (3.12).

Now, using the relations

$$
\partial_{t_{k}} p(z)=-\frac{\partial_{t_{k}} z(p)}{\partial_{p} z(p)}, \quad k \geq 1
$$

between the partial derivatives, and, as a consequence,

$$
\partial_{p} z(p) D\left(z_{1}\right) p(z)=-D\left(z_{1}\right) z(p)
$$

(the derivative in the r.h.s. is taken at "constant $p$ ", namely, we differentiate the coefficients in (3.10) $)$, we can rewrite (3.7) in the form

$$
\partial_{p} z(p) D\left(z_{1}\right) p(z)=-D\left(z_{1}\right) z(p)=\frac{\partial_{t_{1}} z(p)+\partial_{t_{1}} p\left(z_{1}\right) \partial_{p} z(p)}{p\left(z_{1}\right)-p(z)}
$$


After simple transformations this equation can be written as

$$
D\left(z_{1}\right) z(p)=\left\{z(p), \log \left(p\left(z_{1}\right)-p\right)\right\}
$$

where

$$
\{f, g\}:=\frac{\partial f}{\partial t_{1}} \frac{\partial g}{\partial p}-\frac{\partial g}{\partial t_{1}} \frac{\partial f}{\partial p}
$$

is the Poisson bracket. Expanding (3.14) in powers of $z_{1}$ and taking into account (3.11), we obtain the hierarchy of Lax equations

$$
\partial_{t_{k}} z(p)=\left\{B_{k}(p), z(p)\right\}=\left\{\left(z^{k}(p)\right)_{\geq 0}, z(p)\right\} .
$$

We see that the conformal map $z(p)$ plays the role of the Lax function.

\subsubsection{The reduction}

For one-variable reduction, the dependence of $p(z)$ on the times is implemented by means of a single variable $\lambda=\lambda(\mathbf{t}): p(z ; \mathbf{t})=p(z, \lambda(\mathbf{t}))$, i.e., instead of the function of infinitely many independent variables $p(z ; \mathbf{t})$ we now deal with a function of two variables $p(z, \lambda)$ in which the variable $\lambda$ depends on all the times. Our goal is to find a possible form of the functions $p(z, \lambda)$ such that it would be consistent with the infinite hierarchy. The reduction is an exceptional, non-generic solution. We will see that the solutions correspond to conformal maps from slit domains.

Using the chain rule of differentiating, we get:

$$
\partial_{t_{1}} p(z)=\partial_{\lambda} p(z, \lambda) \cdot \partial_{t_{1}} \lambda, \quad D\left(z_{2}\right) p\left(z_{1}\right)=\partial_{\lambda} p\left(z_{1}, \lambda\right) \cdot D\left(z_{2}\right) \lambda .
$$

We also have

$$
D(z) \lambda=D(z) u \cdot \frac{\partial \lambda}{\partial u}=\frac{D(z) u}{\partial_{\lambda} u}=-\frac{\partial_{\lambda} p(z, \lambda)}{\partial_{\lambda} u} \partial_{t_{1}} \lambda,
$$

where we have used equation (3.9) at the last step. Substituting all this into (3.7) and assuming that $\partial_{t_{1}} \lambda \neq 0$, we get

$$
\partial_{\lambda} p\left(z_{1}\right) \partial_{\lambda} p\left(z_{2}\right)=\frac{\partial_{\lambda} p\left(z_{1}\right)-\partial_{\lambda} p\left(z_{2}\right)}{p\left(z_{1}\right)-p\left(z_{2}\right)} \partial_{\lambda} u,
$$

or, after rearranging,

$$
p\left(z_{1}\right)+\frac{\partial_{\lambda} u}{\partial_{\lambda} p\left(z_{1}\right)}=p\left(z_{2}\right)+\frac{\partial_{\lambda} u}{\partial_{\lambda} p\left(z_{2}\right)} .
$$

It follows from this equation that

$$
\xi(\lambda):=p(z)+\frac{\partial_{\lambda} u}{\partial_{\lambda} p(z)}
$$

does not depend on $z$. This equation can be written as the differential equation

$$
\partial_{\lambda} p(z, \lambda)=-\frac{\partial_{\lambda} u}{p(z, \lambda)-\xi(\lambda)}
$$


for the function $p(z, \lambda)$ with arbitrary function $\xi(\lambda)$. It is called the driving function. This function determines the type of the reduction. In particular, if one can put $\lambda=u$, then the equation simplifies:

$$
\partial_{u} p(z, u)=-\frac{1}{p(z, u)-\xi(u)} .
$$

All the coefficients $u_{k}$ in (3.5) become functions of $u$.

Equation (3.18) is the (chordal) Löwner equation for a one-parameter family of conformal maps $p(z, \lambda)$ from the upper half plane with a curved slit starting at the real axis to the upper half plane. Here $z$ is the coordinate in the upper half plane with a slit and $\lambda$ is the parameter of the curved slit. As we have seen in section 2.1, the form of the slit is determined by the function $\xi(\lambda)$, the variable $\lambda$ being a parameter along the curve (for example, the length of the curve). One can say, therefore, that the type of the reduction is determined by the form of the slit. The choice $\lambda=u$ corresponds to the choice of capacity as a natural parameter along the curved slit.

We see that one-variable reductions of the dKP hierarchy are determined by the form of the slit if it comes from such a family of conformal maps and are given by solutions of the chordal Löwner equation (3.18).

The differential equation (3.18) can be equivalently written as a partial differential equation for the Lax function $z(p)$ which maps the upper half plane to the upper half plane with a slit. Indeed, since

$$
\partial_{\lambda} p(z)=-\frac{\partial_{\lambda} z(p)}{\partial_{p} z(p)}
$$

we represent equation (3.18) in the equivalent form

$$
\frac{\partial z(p)}{\partial \lambda}=\frac{\partial_{\lambda} u}{p-\xi(\lambda)} \frac{\partial z(p)}{\partial p}
$$

which is also referred to as chordal Löwner equation. We see that the Lax function satisfies the chordal Löwner equation of this type.

Given a one-variable reduction, it is not difficult to obtain the solution to the hierarchy. Using (3.9), we have:

$$
D(z) \lambda=D(z) u \cdot \partial_{u} \lambda=-\partial_{t_{1}} p(z) \partial_{u} \lambda=-\partial_{\lambda} p(z) \partial_{t_{1}} \lambda \partial_{u} \lambda=\frac{\partial_{t_{1}} \lambda}{p(z)-\xi(\lambda)},
$$

where we have used the Löwner equation (3.18) at the last step. Differentiating (3.11) with respect to $p$, we can write

$$
D(z) \lambda=\sum_{k \geq 1} \frac{z^{-k}}{k} B_{k}^{\prime}(\xi(\lambda)) \partial_{t_{1}} \lambda
$$

where $B_{k}^{\prime}(p)=\partial_{p} B_{k}(p)$ is the derivative of the Faber polynomial, or

$$
\partial_{t_{k}} \lambda=B_{k}^{\prime}(\xi(\lambda)) \partial_{t_{1}} \lambda, \quad k \geq 1
$$


which is a one-component differential equation of the hydrodynamic type. The implicit solution for $\lambda=\lambda(\mathbf{t})$ of the hodograph relation

$$
t_{1}+\sum_{k \geq 2} t_{k} B_{k}^{\prime}(\xi(\lambda))=R(\lambda)
$$

gives a solution of (3.21). Here $R(\lambda)$ is an arbitrary function.

\subsection{One-variable reductions of the dBKP hierarchy}

\subsubsection{The dBKP hierarchy}

For the theory of the dBKP hierarchy see [37, 38, 39]. Let $\mathbf{t}=\left\{t_{1}, t_{3}, t_{5}, \ldots\right\}$ be an infinite set of independent variables ("times") indexed by odd natural numbers. Here we suppose that they are real. It is convenient to introduce the differential operator

$$
D^{\mathrm{o}}(z)=\sum_{k \geq 1, \text { odd }} \frac{z^{-k}}{k} \partial_{t_{k}} .
$$

The dBKP hierarchy in the Hirota form can be written as the equation

$$
1-2 \frac{D^{\circ}\left(z_{1}\right) \partial_{t_{1}} F-D^{\circ}\left(z_{2}\right) \partial_{t_{1}} F}{z_{1}-z_{2}}=\left(1-2 \frac{D^{\circ}\left(z_{1}\right) \partial_{t_{1}} F+D^{\circ}\left(z_{2}\right) \partial_{t_{1}} F}{z_{1}+z_{2}}\right) e^{4 D^{\circ}\left(z_{1}\right) D^{\circ}\left(z_{2}\right) F}
$$

for the real-valued function $F=F(\mathbf{t})$ which should be valid for any $z_{1}, z_{2}$. In terms of the (odd) function

$$
p(z)=z-2 D^{\circ}(z) \partial_{t_{1}} F, \quad p(-z)=-p(z)
$$

the equation (3.24) reads

$$
\frac{p\left(z_{1}\right)-p\left(z_{2}\right)}{z_{1}-z_{2}}=\frac{p\left(z_{1}\right)+p\left(z_{2}\right)}{z_{1}+z_{2}} e^{4 D^{\circ}\left(z_{1}\right) D^{\circ}\left(z_{2}\right) F}
$$

The expansion of the function $p(z)$ in the Laurent series is

$$
p(z)=z-\frac{u}{z}+\sum_{k \geq 3, \text { odd }} \frac{u_{k}}{z^{k}},
$$

where

$$
u=u(\mathbf{t})=2 \partial_{t_{1}}^{2} F .
$$

Taking logarithm of equation (3.26), differentiating with respect to $t_{1}$ and using the definition (3.25), we obtain the equation

$$
2 D^{\circ}\left(z_{1}\right) p\left(z_{2}\right)=\partial_{t_{1}} \log \frac{p\left(z_{1}\right)+p\left(z_{2}\right)}{p\left(z_{1}\right)-p\left(z_{2}\right)} \text {. }
$$

from which it follows that $D^{\mathrm{o}}\left(z_{1}\right) p\left(z_{2}\right)=D^{\mathrm{o}}\left(z_{2}\right) p\left(z_{1}\right)$ (this follows also from the definition (3.25)). Tending $z_{2} \rightarrow \infty$, we get

$$
\partial_{t_{1}} p(z)=-D^{\circ}(z) u .
$$


Let us rewrite equation (3.29) in terms of the function $z(p)$, inverse to the $p(z)$ (like $p(z)$, it is an odd function with the Laurent series of the form $\left.z(p)=p+O\left(p^{-1}\right)\right)$. The calculation is similar to the one leading to equation (3.14). Using the relation (3.13), we get, after simple transformations:

$$
2 D^{\circ}\left(z_{1}\right) z(p)=\left\{z(p), \log \frac{p\left(z_{1}\right)-p}{p\left(z_{1}\right)+p}\right\}
$$

where $\{$,$\} is the Poisson bracket (3.15). This is the generating Lax equation for the$ $\mathrm{dBKP}$ hierarchy, $z(p)$ being the Lax function. Expanding equation (3.31) in powers of $z_{1}$, one obtains the hierarchy of Lax equations through the Faber polynomials. The Faber polynomials $B_{k}(p)$ are introduced by the same formulas (3.11), (3.12) as in section 3.1.1. The fact that $p(z)$ is an odd function implies that $B_{k}(-p)=(-1)^{k} B_{k}(p)$ and we have the expansion

$$
\log \frac{p(z)+p}{p(z)-p}=2 \sum_{k \geq 1, \text { odd }} \frac{z^{-k}}{k} B_{k}(p), \quad B_{k}(p)=\left(z^{k}(p)\right)_{\geq 0} .
$$

The Lax equations are of the form (3.16).

We note that the dispersionless limit of the CKP hierarchy is known to be the same as for the BKP hierarchy (but the dispersionful hierarhies are different), so it is given by the same equation (3.24).

\subsubsection{The reduction}

The definition of the one-variable reduction is the same as in section 3.1.2. Substituting the reduction condition into equation (3.29), we get:

$$
\partial_{\lambda} p\left(z_{1}\right) D^{\mathrm{o}}\left(z_{2}\right) \lambda=\frac{p\left(z_{1}\right) \partial_{\lambda} p\left(z_{2}\right)-p\left(z_{2}\right) \partial_{\lambda} p\left(z_{1}\right)}{p^{2}\left(z_{1}\right)-p^{2}\left(z_{2}\right)} \partial_{t_{1}} \lambda
$$

Next, plugging here the relation

$$
D^{\mathrm{o}}\left(z_{2}\right) \lambda=-\frac{\partial_{\lambda} p\left(z_{2}\right)}{\partial_{\lambda} u} \partial_{t_{1}} \lambda
$$

(a consequence of (3.30) and the reduction condition) and assuming that $\partial_{t_{1}} \lambda$ is not identically zero, we obtain:

$$
\partial_{\lambda} p\left(z_{1}\right) \partial_{\lambda} p\left(z_{2}\right)=\frac{p\left(z_{2}\right) \partial_{\lambda} p\left(z_{1}\right)-p\left(z_{1}\right) \partial_{\lambda} p\left(z_{2}\right)}{p^{2}\left(z_{1}\right)-p^{2}\left(z_{2}\right)} \partial_{\lambda} u
$$

or, after, rearranging,

$$
p^{2}\left(z_{1}\right)+\frac{p\left(z_{1}\right) \partial_{\lambda} u}{\partial_{\lambda} p\left(z_{1}\right)}=p^{2}\left(z_{2}\right)+\frac{p\left(z_{2}\right) \partial_{\lambda} u}{\partial_{\lambda} p\left(z_{2}\right)} .
$$

It follows from this relation that

$$
\xi^{2}(\lambda):=p^{2}(z)+\frac{p(z) \partial_{\lambda} u}{\partial_{\lambda} p(z)}
$$


does not depend on $z$. This is equivalent to the differential equation

$$
\partial_{\lambda} p(z)=-\frac{p(z) \partial_{\lambda} u}{p^{2}(z)-\xi^{2}(\lambda)}
$$

or

$$
\partial_{\lambda} p(z)=-\frac{\partial_{\lambda} u / 2}{p(z)-\xi(\lambda)}-\frac{\partial_{\lambda} u / 2}{p(z)+\xi(\lambda)}
$$

The corresponding equation for the inverse function is

$$
\partial_{\lambda} z(p)=\frac{p \partial_{\lambda} u}{p^{2}-\xi^{2}(\lambda)} \partial_{p} z(p)
$$

This is the quadrant Löwner equation [10] with the driving function $\xi(\lambda)$. Suitably normalized families of conformal maps of slit domains in the quadrant are solutions to this equation.

Using the quadrant Löwner equation, we can write

$$
D^{\circ}(z) \lambda=\frac{1}{2}\left(\frac{1}{p(z)-\xi(\lambda)}+\frac{1}{p(z)+\xi(\lambda)}\right) \partial_{t_{1}} \lambda
$$

Using the expansion (3.32), we obtain from here the system of partial differential equations for $\lambda$ of the hydrodynamic type:

$$
\partial_{t_{k}} \lambda=B_{k}^{\prime}(\xi(\lambda)) \partial_{t_{1}} \lambda, \quad k=1,3,5, \ldots
$$

with the general solution in the hodograph form

$$
t_{1}+\sum_{k \geq 3, \text { odd }} B_{k}^{\prime}(\xi(\lambda))=R(\lambda)
$$

where $R(\lambda)$ is an arbitrary function.

\subsection{One-variable reductions of the dToda hierarchy}

\subsubsection{The dToda hierarchy}

For the dToda hierarchy, there are two sets of infinitely many independent variables ("times"): complex variables $\mathbf{t}=\left\{t_{1}, t_{2}, t_{3}, \ldots\right\}$ and $\overline{\mathbf{t}}=\left\{\bar{t}_{1}, \bar{t}_{2}, \bar{t}_{3}, \ldots\right\}$. Here we take $\overline{\mathbf{t}}$ not independent from $\mathbf{t}$ but assume that they are complex conjugate to $\mathbf{t}$. There is also a real variable $t_{0}$. Similarly to (3.1), we introduce the differential operators

$$
D(z)=\sum_{k \geq 1} \frac{z^{-k}}{k} \partial_{t_{k}}, \quad \bar{D}(\bar{z})=\sum_{k \geq 1} \frac{\bar{z}^{-k}}{k} \partial_{\bar{t}_{k}} .
$$

The dToda hierarchy can be written as the following system of equations:

$$
\left\{\begin{array}{l}
\left(z_{1}-z_{2}\right) e^{D\left(z_{1}\right) D\left(z_{2}\right) F}=z_{1} e^{-\partial_{t_{0}} D\left(z_{1}\right) F}-z_{2} e^{-\partial_{t_{0}} D\left(z_{2}\right) F}, \\
z_{1} \bar{z}_{2}\left(1-e^{-D\left(z_{1}\right) \bar{D}\left(\bar{z}_{2}\right) F}\right)=e^{\partial_{t_{0}}\left(\partial_{t_{0}}+D\left(z_{1}\right)+\bar{D}\left(\bar{z}_{2}\right)\right) F}
\end{array}\right.
$$


for a real-valued function $F=F\left(\mathbf{t}, t_{0}, \overline{\mathbf{t}}\right)$, which should hold for any $z_{1}, z_{2}$. Besides, there is the equation which is complex-conjugate to the first equation in (3.39). It is convenient to introduce the function

$$
w(z)=z \exp \left(-\frac{1}{2} \partial_{t_{0}}^{2} F-\partial_{t_{0}} D(z) F\right)
$$

then the equations of the dToda hierarchy can be written in the form

$$
\left\{\begin{array}{l}
\frac{w\left(z_{1}\right)-w\left(z_{2}\right)}{z_{1}-z_{2}}=e^{-\frac{1}{2} \partial_{t_{0}}^{2} F+D\left(z_{1}\right) D\left(z_{2}\right) F} \\
1-\left(w\left(z_{1}\right) \bar{w}\left(\bar{z}_{2}\right)\right)^{-1}=e^{-D\left(z_{1}\right) \bar{D}\left(\bar{z}_{2}\right) F}
\end{array}\right.
$$

In the second equation $\bar{w}(\bar{z})=\overline{w(z)}$. The function $w(z)$ depends also on all the times: $w(z)=w\left(z ; \mathbf{t}, t_{0}, \overline{\mathbf{t}}\right)$. The expansion of the function $w(z)$ into the series has the form

$$
w(z)=\frac{z}{r}+\sum_{k \geq 0} \frac{u_{k}}{z^{k}}
$$

where $r$ is a real quantity given by

$$
\log r=\frac{1}{2} \partial_{t_{0}}^{2} F
$$

Note that

$$
D(z) \log r=\frac{1}{2} \partial_{t_{0}}^{2} D(z) F=-\frac{1}{2} \partial_{t_{0}} \log (r w(z)) .
$$

Another way to derive equation (3.44) is as follows. Consider the first equation in (3.41). Let us write it in the form

$$
e^{\left(\partial_{t_{0}}+D\left(z_{1}\right)\right)\left(\partial_{t_{0}}+D\left(z_{2}\right)\right) F}=\frac{r w^{-1}\left(z_{1}\right)-r w^{-1}\left(z_{2}\right)}{z_{1}^{-1}-z_{2}^{-1}} .
$$

Note that this equation already contains the definition of the function $w(z)(\underline{3.40})$ as the limit case $z_{2} \rightarrow \infty$. Let us take logarithm of the both sides of (3.45) and differentiate with respect to $t_{0}$. Taking into account (3.40), we get:

$$
\left(\partial_{t_{0}}+D\left(z_{2}\right)\right) \log \frac{w\left(z_{1}\right)}{r z_{1}}=-\frac{\partial_{t_{0}}\left(r w^{-1}\left(z_{1}\right)\right)-\partial_{t_{0}}\left(r w^{-1}\left(z_{2}\right)\right)}{r\left(w^{-1}\left(z_{1}\right)-w^{-1}\left(z_{2}\right)\right)} .
$$

The right hand side is symmetric with respect to permutation of $z_{1}, z_{2}$, whence

$$
\left(\partial_{t_{0}}+D\left(z_{2}\right)\right) \log \left(r w^{-1}\left(z_{1}\right)\right)=\left(\partial_{t_{0}}+D\left(z_{1}\right)\right) \log \left(r w^{-1}\left(z_{2}\right)\right) .
$$

Tending $z_{2} \rightarrow \infty$, we get, using (3.42), that

$$
\partial_{t_{0}} \log \left(r w^{-1}(z)\right)=\left(\partial_{t_{0}}+D(z)\right) \log r^{2}
$$

which is the same as (3.44).

In what follows we assume that the series (3.42) converges in a neighborhood of infinity and defines there a meromorphic function. This function $w(z)$ has the meaning of the 
conformal map of a domain containing $\infty$ to the exterior of the unit circle normalized in such a way that $w(\infty)=\infty$ and $\arg w^{\prime}(\infty)>0$. The times $t_{k}, \bar{t}_{k}$ are harmonic moments of the (exterior of) the domain and $t_{0}$ is proportional to its area [2, 40]. The quantity $r>0$ is called the conformal radius of the domain.

Taking logarithm of equations (3.41), differentiating with respect to $t_{0}$ and using (3.44), we obtain the equations

$$
\begin{aligned}
& D\left(z_{1}\right) \log w(z)=-\partial_{t_{0}} \log \left(w\left(z_{1}\right)-w(z)\right)+\frac{1}{2} \partial_{t_{0}} \log \left(z_{1} w\left(z_{1}\right) / r\right) \\
& \bar{D}\left(\bar{z}_{1}\right) \log w(z)=\partial_{t_{0}} \log \left(\bar{w}\left(\bar{z}_{1}\right)-w^{-1}(z)\right)-\frac{1}{2} \partial_{t_{0}} \log \left(\bar{z}_{1} \bar{w}\left(\bar{z}_{1}\right) / r\right) .
\end{aligned}
$$

It is instructive to rewrite them in terms of the inverse function to $w(z)$ which we denote as $z(w)$ and the analytic continuation $\bar{z}\left(w^{-1}\right)$ of the complex conjugate function $\overline{z(w)}$ from the unit circle. They have the expansions

$$
z(w)=r w+\sum_{k \geq 0} v_{k} w^{-k}, \quad \bar{z}\left(w^{-1}\right)=r w^{-1}+\sum_{k \geq 0} \bar{v}_{k} w^{k} .
$$

Noting the relations

$$
\partial_{t_{k}} w(z)=-\frac{\partial_{t_{k}} z(w)}{\partial_{w} z(w)}, \quad k \geq 0
$$

between the partial derivatives, and, as a consequence,

$$
w \partial_{w} z(w) D\left(z_{1}\right) \log w(z)=-D\left(z_{1}\right) z(w)
$$

(the derivative in the r.h.s. is taken at constant $w$ ), we can rewrite the first equation in (3.49) in the form

$$
\frac{D\left(z_{1}\right) z(w)}{w \partial_{w} z(w)}=\frac{\partial_{t_{0}} w\left(z_{1}\right)+\partial_{t_{0}} z(w) / \partial_{w} z(w)}{w\left(z_{1}\right)-w(z)}-\frac{1}{2} \partial_{t_{0}} \log \left(z_{1} w\left(z_{1}\right) / r\right)
$$

or

$$
D\left(z_{1}\right) z(w)=\left\{z(w), \log \left(w\left(z_{1}\right)-w\right)+\frac{1}{2} \log \frac{r}{z_{1} w\left(z_{1}\right)}\right\}_{\text {Toda }},
$$

where

$$
\{f, g\}_{\text {Toda }}=w \frac{\partial f}{\partial w} \frac{\partial g}{\partial t_{0}}-w \frac{\partial f}{\partial t_{0}} \frac{\partial g}{\partial w}
$$

is the Poisson bracket for the dToda hierarchy. Similarly, the second equation in (3.49) can be written in the form

$$
\bar{D}\left(\bar{z}_{1}\right) z(w)=-\left\{z(w), \log \left(\bar{w}\left(\bar{z}_{1}\right)-w^{-1}\right)+\frac{1}{2} \log \frac{r}{\bar{z}_{1} \bar{w}\left(\bar{z}_{1}\right)}\right\}_{\text {Toda }} .
$$

Equations (3.51), (3.53) are generating Lax equations for the dToda hierarchy. To see this, let us introduce the Faber polynomials $A_{k}(w), \bar{A}_{k}\left(w^{-1}\right)$ according to the expansions

$$
\begin{aligned}
& -\log (w(z)-w)+\frac{1}{2} \log \frac{z w(z)}{r}=\sum_{k \geq 1} \frac{z^{-k}}{k} A_{k}(w), \\
& -\log \left(\bar{w}(\bar{z})-w^{-1}\right)+\frac{1}{2} \log \frac{\bar{z} \bar{w}(z)}{r}=\sum_{k \geq 1} \frac{\bar{z}^{-k}}{k} \bar{A}_{k}\left(w^{-1}\right),
\end{aligned}
$$


then equations (3.51), (3.53) imply the Lax equations of the dToda hierarchy:

$$
\partial_{t_{k}} z(w)=\left\{A_{k}(w), z(w)\right\}_{\text {Toda }}, \quad \partial_{\bar{t}_{k}} z(w)=-\left\{\bar{A}_{k}\left(w^{-1}\right), z(w)\right\}_{\text {Toda }} .
$$

It is easy to see that

$$
A_{k}(w)=\left(z^{k}(w)\right)_{>0}+\frac{1}{2}\left(z^{k}(w)\right)_{0}, \quad \bar{A}_{k}\left(w^{-1}\right)=\left(\bar{z}^{k}\left(w^{-1}\right)\right)_{<0}+\frac{1}{2}\left(\bar{z}^{k}\left(w^{-1}\right)\right)_{0},
$$

where $(\ldots)_{>0},(\ldots)_{<0},(\ldots)_{0}$ mean respectively positive, negative and constant parts of a Laurent series. To see this, we fix a point $w_{1}=z\left(w_{1}\right)$ and write

$$
\log \left(1-\frac{z(w)}{z\left(w_{1}\right)}\right)=\log \left(1-\frac{w}{w\left(z_{1}\right)}\right)+\log \frac{r w\left(z_{1}\right)}{z_{1}}+\log \frac{z\left(w_{1}\right)-z(w)}{r\left(w_{1}-w\right)} .
$$

Now, we notice that the expansion of the first (third) term contains only positive (negative) powers of $w$ while the rest is just the constant term. Equations (3.56) easily follow from this.

\subsubsection{The reduction}

The one-variable reduction is defined similarly to the case of the dKP hierarchy. For the one-variable reduction, the dependence of $w(z)$ on all the times is implemented by a single function $\lambda=\lambda\left(\mathbf{t}, t_{0}, \overline{\mathbf{t}}\right): w\left(z ; \mathbf{t}, t_{0}, \overline{\mathbf{t}}\right)=w\left(z, \lambda\left(\mathbf{t}, t_{0}, \overline{\mathbf{t}}\right)\right)$, i.e., instead of a function of infinitely many variables $w\left(z ; \mathbf{t}, t_{0}, \overline{\mathbf{t}}\right)$ we deal with a function of two variables $w(z, \lambda)$, where the variable $\lambda$ is a function of all the times. Here we again assume that the formal series $w(z, \lambda)$ gives a one-parameter family of functions in some domain in the exterior of the unit disk. Our goal is to find a possible form of the functions $w(z, \lambda)$ such that it would be consistent with the dToda hierarchy.

In the calculations below we closely follow [9, 41]. Using the chain rule of differentiating, we have:

$$
\begin{aligned}
\partial_{t_{0}}\left(r w^{-1}(z)\right) & =\partial_{\lambda}\left(r w^{-1}(z, \lambda)\right) \cdot \partial_{t_{0}} \lambda \\
\left(\partial_{t_{0}}+D\left(z_{2}\right)\right) \log \left(r w^{-1}\left(z_{1}\right)\right) & =\partial_{\lambda} \log \left(r w^{-1}\left(z_{1}, \lambda\right)\right) \cdot\left(\partial_{t_{0}}+D\left(z_{2}\right)\right) \lambda
\end{aligned}
$$

and

$$
\begin{gathered}
\left(\partial_{t_{0}}+D(z)\right) \lambda=\left(\partial_{t_{0}}+D(z)\right) \log r \cdot \frac{\partial \lambda}{\partial \log r} \\
=\frac{\left(\partial_{t_{0}}+D(z)\right) \log r}{\partial_{\lambda} \log r}=\frac{\partial_{\lambda} \log \left(r w^{-1}(z, \lambda)\right)}{2 \partial_{\lambda} \log r} \partial_{t_{0}} \lambda
\end{gathered}
$$

where we have used (3.48) in the last step. Substituting this into (3.46) and assuming that $\partial_{t_{0}} \lambda \neq 0$, we get:

$$
\partial_{\lambda} \log \left(r w^{-1}\left(z_{1}\right)\right) \partial_{\lambda} \log \left(r w^{-1}\left(z_{2}\right)\right)=2 \frac{\partial_{\lambda}\left(r w^{-1}\left(z_{1}\right)\right)-\partial_{\lambda}\left(r w^{-1}\left(z_{2}\right)\right)}{r w^{-1}\left(z_{1}\right)-r w^{-1}\left(z_{2}\right)} \partial_{\lambda} \log r
$$

or, after rearranging,

$$
-w\left(z_{1}\right)+\frac{2 w\left(z_{1}\right) \partial_{\lambda} \log r}{\partial_{\lambda} \log \left(r w^{-1}\left(z_{1}\right)\right)}=-w\left(z_{2}\right)+\frac{2 w\left(z_{2}\right) \partial_{\lambda} \log r}{\partial_{\lambda} \log \left(r w^{-1}\left(z_{2}\right)\right)} .
$$


It follows from this equation that

$$
\eta(\lambda):=w(z)-\frac{2 w(z) \partial_{\lambda} \log r}{\partial_{\lambda} \log \left(r w^{-1}(z)\right)}=-w(z) \frac{\partial_{\lambda} \log (r w(z))}{\partial_{\lambda} \log \left(r w^{-1}(z)\right)}
$$

does not depend on $z$. This relation can be rewritten as the differential equation

$$
\partial_{\lambda} \log w(z, \lambda)=-\frac{w(z, \lambda)+\varphi(\lambda)}{w(z, \lambda)-\eta(\lambda)} \partial_{\lambda} \log r
$$

for the function $w(z, \lambda)$ with an arbitrary function $\eta(\lambda)$. In particular, one can put $\lambda=\log r$, then the equation simplifies:

$$
\frac{\partial \log w(z)}{\partial \log r}=-\frac{w(z)+\eta(r)}{w(z)-\eta(r)}
$$

and all the coefficients $u_{k}$ in (3.42) become functions of $r$.

Let us now see what the second equation in (3.41) gives. We write it in the form

$$
\log \left(w\left(z_{1}\right) \overline{w\left(z_{2}\right)}-1\right)=z_{1} \bar{z}_{2}-\left(\partial_{t_{0}}+D\left(z_{1}\right)\right)\left(\partial_{t_{0}}+\bar{D}\left(\bar{z}_{2}\right)\right) F
$$

and differentiate both sides with respect to $t_{0}$. We obtain:

$$
\frac{\partial_{t_{0}} w\left(z_{1}\right) \overline{w\left(z_{2}\right)}+\partial_{t_{0}} \overline{w\left(z_{2}\right)} w\left(z_{1}\right)}{w\left(z_{1}\right) \overline{w\left(z_{2}\right)}-1}=-\left(\partial_{t_{0}}+D\left(z_{1}\right)\right) \log \left(r \overline{w^{-1}\left(z_{2}\right)}\right) .
$$

Substituting here the reduction condition and using the relations obtained above, we get:

$$
2 \partial_{\lambda} \log r \frac{\partial_{\lambda} w\left(z_{1}\right) \overline{w\left(z_{2}\right)}+\partial_{\lambda} \overline{w\left(z_{2}\right)} w\left(z_{1}\right)}{w\left(z_{1}\right) \overline{w\left(z_{2}\right)}-1}=\partial_{\lambda} \log \left(r w^{-1}\left(z_{1}\right)\right) \partial_{\lambda} \log \left(r \overline{w^{-1}\left(z_{2}\right)}\right) .
$$

After simple transformations this equation can be represented in the form

$$
\left(w\left(z_{1}\right)-\frac{2 w\left(z_{1}\right) \partial_{\lambda} \log r}{\partial_{\lambda} \log \left(r w^{-1}\left(z_{1}\right)\right.}\right)\left(\overline{w\left(z_{2}\right)}-\frac{2 \overline{w\left(z_{2}\right)} \partial_{\lambda} \log r}{\partial_{\lambda} \log \left(r \overline{w^{-1}\left(z_{2}\right)}\right.}\right)=1 .
$$

The definition (3.57) implies that this equation means that

$$
\eta(\lambda) \overline{\eta(\lambda)}=|\eta(\lambda)|^{2}=1
$$

i.e., $\eta(\lambda)$ has the form

$$
\eta(\lambda)=e^{i \xi(\lambda)}
$$

with a real-valued function $\xi(\lambda)$.

Therefore, our differential equation acquires the form

$$
\partial_{\lambda} w(z, \lambda)=-w(z, \lambda) \frac{w(z, \lambda)+e^{i \xi(\lambda)}}{w(z, \lambda)-e^{i \xi(\lambda)}} \partial_{\lambda} \log r
$$

The equation (3.64) is the (radial) Löwner equation (2.20) for the conformal map w(z) from the exterior of the unit disk with a slit of arbitrary form to the exterior of the unit 
disk. The form of the slit is parametrized by the driving function $\xi(\lambda)$ which simultaneously determines the type of the reduction. Note that in (2.20) we assumed that $r=e^{t}$. The last factor $\partial_{\lambda} \log r$ comes from a different parametrization.

Introducing $p(z, \lambda)=-i \log w(z, \lambda)$, one can write the radial Löwner equation in the form

$$
\partial_{\lambda} p(z, \lambda)=\cot \left(\frac{p(z, \lambda)-\xi(\lambda)}{2}\right) \partial_{\lambda} \log r
$$

which is the trigonometric extension of the chordal (rational) Löwner equation (3.18). The function $p(z, \lambda)$ maps a half-strip with a slit onto the half-strip.

Note also that equation (3.64) can be equivalently rewritten as the partial differential equation

$$
\partial_{\lambda} z(w)=\frac{w+e^{i \xi(\lambda)}}{w-e^{i \xi(\lambda)}} \partial_{\lambda} \log r w \partial_{w} z(w)
$$

for the Lax function $z(w)$ (the inverse of $w(z)$ ).

The dependence of $\lambda$ on the times $t_{k}, \bar{t}_{k}$ is determined by a system of equations of the hydrodynamic type. They follow from equation (3.44) which can be written as

$$
D(z) \lambda=\frac{D(z) \log r}{\partial_{\lambda} \log r}=-\frac{1}{2}\left(1+\frac{\partial_{\lambda} \log w(z)}{\partial_{\lambda} \log r}\right) \partial_{t_{0}} \lambda,
$$

taking into account the reduction condition. Using the Löwner equation (3.64), we obtain:

$$
D(z) \lambda=\frac{\eta(\lambda) \partial_{t_{0}} \lambda}{w(z)-\eta(\lambda)}
$$

From (3.54) we conclude that

$$
\frac{\eta}{w(z)-\eta}=\sum_{k \geq 1} \frac{z^{-k}}{k} \phi_{k}(\eta), \quad \phi_{k}(w)=w \partial_{w} A_{k}(w)
$$

Therefore, the system of hydrodynamic type reads

$$
\partial_{t_{k}} \lambda=\phi_{k}(\eta(\lambda)) \partial_{t_{0}} \lambda
$$

Equations containing $\bar{t}_{k}$-derivatives are obtained by complex conjugation of (3.68). The hodograph equation

$$
t_{0}+\sum_{k \geq 1} t_{k} \phi_{k}(\eta(\lambda))+\sum_{k \geq 1} \bar{t}_{k} \overline{\phi_{k}(\eta(\lambda))}=R(\lambda),
$$

gives solutions in an implicit form. Here $R(\lambda)$ is an arbitrary function.

\subsection{One-variable reductions of the dDKP hierarchy}

The DKP hierarchy (known also as Pfaff-KP) is an integrable hierarchy with $D_{\infty}$ symmetry. It was first introduced by M.Jimbo and T.Miwa in 1983 [42] and it was subsequently rediscovered under the names coupled KP hierarchy [43] and the Pfaff lattice [44, 45], see also [46, 47, 48. The solutions and the algebraic structure were studied in [49, 50, 51, the relation to matrix integrals was elaborated in [44, 45, 46, 52, 53]. 


\subsubsection{Algebraic form of the dDKP hierarchy}

The dispersionless version of the DKP hierarchy (the dDKP hierarchy) was suggested in [54, 55]. It is an infinite system of differential equations for a function $F=F(\mathbf{t})$ of the infinite number of "times" $\mathbf{t}=\left\{t_{0}, t_{1}, t_{2}, \ldots\right\}$. Here again we assume that $F$ is a realvalued function of real variables $\mathbf{t}$. The differential equations are obtained by expanding equations

$$
\begin{gathered}
e^{D\left(z_{1}\right) D\left(z_{2}\right) F}\left(1-\frac{1}{z_{1}^{2} z_{2}^{2}} e^{2 \partial_{t_{0}}\left(2 \partial_{t_{0}}+D\left(z_{1}\right)+D\left(z_{2}\right)\right) F}\right)=1-\frac{\partial_{t_{1}} D\left(z_{1}\right) F-\partial_{t_{1}} D\left(z_{2}\right) F}{z_{1}-z_{2}} \\
e^{-D\left(z_{1}\right) D\left(z_{2}\right) F} \frac{z_{1}^{2} e^{-2 \partial_{t_{0}} D\left(z_{1}\right) F}-z_{2}^{2} e^{-2 \partial_{t_{0}} D\left(z_{2}\right) F}}{z_{1}-z_{2}}=z_{1}+z_{2}-\partial_{t_{1}}\left(2 \partial_{t_{0}}+D\left(z_{1}\right)+D\left(z_{2}\right)\right) F
\end{gathered}
$$

where $D(z)$ is the operator (3.1), in powers of $z_{1}, z_{2}$.

In this section we use the differential operator

$$
\nabla(z)=\partial_{t_{0}}+D(z)
$$

which in the dDKP case is more convenient than $D(z)$. Introducing the functions

$$
p(z)=z-\partial_{t_{1}} \nabla(z) F, \quad w(z)=z^{2} e^{-2 \partial_{t_{0}} \nabla(z) F},
$$

we can rewrite equations (3.70), (3.71) in a more compact form

$$
\begin{gathered}
e^{D\left(z_{1}\right) D\left(z_{2}\right) F}\left(1-\frac{1}{w\left(z_{1}\right) w\left(z_{2}\right)}\right)=\frac{p\left(z_{1}\right)-p\left(z_{2}\right)}{z_{1}-z_{2}}, \\
e^{-D\left(z_{1}\right) D\left(z_{2}\right) F+2 \partial_{t_{0}}^{2} F} \frac{w\left(z_{1}\right)-w\left(z_{2}\right)}{z_{1}-z_{2}}=p\left(z_{1}\right)+p\left(z_{2}\right) .
\end{gathered}
$$

Multiplying the two equations, we observe that $e^{D\left(z_{1}\right) D\left(z_{2}\right) F}$ cancels and we get the relation

$$
p^{2}\left(z_{1}\right)-e^{2 F_{00}}\left(w\left(z_{1}\right)+w^{-1}\left(z_{1}\right)\right)=p^{2}\left(z_{2}\right)-e^{2 F_{00}}\left(w\left(z_{2}\right)+w^{-1}\left(z_{2}\right)\right)
$$

from which it follows that $p^{2}(z)-e^{2 F_{00}}\left(w(z)+w^{-1}(z)\right)$ does not depend on $z$ (here and below we use the short-hand notation $F_{m n}=\partial_{t_{m}} \partial_{t_{n}} F$ ). Tending $z$ to infinity, we find that this expression is equal to $F_{02}-2 F_{11}-F_{01}^{2}$. Therefore, we conclude that the functions $p(z), w(z)$ satisfy the algebraic equation [55]

$$
p^{2}(z)=R^{2}\left(w(z)+w^{-1}(z)\right)+V
$$

where

$$
R=e^{F_{00}}, \quad V=F_{02}-2 F_{11}-F_{01}^{2}
$$

are real numbers depending on the times ( $R$ is positive). The equation ( $(3.76)$ defines a family of elliptic curves, parametrized by $R$ and $V$, with $p, w$ being algebraic functions on the curve. 


\subsubsection{Elliptic form of the dDKP hierarchy}

Remarkably, the dDKP hierarchy admits an elliptic reformulation suggested in [11], see also [32, 33]. The first step in this reformulation is uniformization of the curve (3.76) through elliptic functions. To this end, we use the standard Jacobi theta functions $\theta_{a}(u)=\theta_{a}(u, \tau)(a=1,2,3,4)$. Their definition is given in Appendix A.

The uniformization of the relation (3.76) is as follows:

$$
p(z)=\gamma \theta_{4}^{2}(0) \frac{\theta_{2}(u(z)) \theta_{3}(u(z))}{\theta_{1}(u(z)) \theta_{4}(u(z))}, \quad w(z)=\left(\frac{\theta_{4}(u(z))}{\theta_{1}(u(z))}\right)^{2},
$$

where $u(z)=u(z, \mathbf{t})$ is some function of $z, \gamma$ is a $z$-independent factor, and

$$
R=\gamma \theta_{2}(0) \theta_{3}(0), \quad V=-\gamma^{2}\left(\theta_{2}^{4}(0)+\theta_{3}^{4}(0)\right) .
$$

In this parametrization, the equation of the curve (3.76) is equivalent to the identity

$$
\theta_{4}^{2}(0) \frac{\theta_{2}^{2}(u) \theta_{3}^{2}(u)}{\theta_{1}^{2}(u) \theta_{4}^{2}(u)}=\theta_{2}^{2}(0) \theta_{3}^{2}(0)\left(\frac{\theta_{4}^{2}(u)}{\theta_{1}^{2}(u)}+\frac{\theta_{1}^{2}(u)}{\theta_{4}^{2}(u)}\right)-\left(\theta_{2}^{4}(0)+\theta_{3}^{4}(0)\right)
$$

which can be proved either by using some standard identities for theta-functions or by comparing analytical properties of the both sides.

Note that $\gamma$ as well as the modular parameter $\tau$ depend on the times: $\gamma=\gamma(\mathbf{t})$, $\tau=\tau(\mathbf{t})$. The reality of the coefficients $R^{2}, V$ implies certain restrictions on possible values of $\tau$. In what follows we assume that $\tau$ is purely imaginary. We normalize the function $u(z)$ by the condition $u(\infty)=0$, with the expansion around $\infty$ being

$$
u(z, \mathbf{t})=\frac{c_{1}(\mathbf{t})}{z}+\sum_{k \geq 2} \frac{c_{k}(\mathbf{t})}{z^{k}}
$$

with real coefficients $c_{k}$.

It is not difficult to check the identity

$$
\frac{w\left(z_{1}\right)-w\left(z_{2}\right)}{p\left(z_{1}\right)+p\left(z_{2}\right)}=-\frac{1}{\gamma \theta_{2}(0) \theta_{3}(0)} \frac{\theta_{4}\left(u_{1}\right) \theta_{4}\left(u_{2}\right)}{\theta_{1}\left(u_{1}\right) \theta_{1}\left(u_{2}\right)} \frac{\theta_{1}\left(u_{1}-u_{2}\right)}{\theta_{4}\left(u_{1}-u_{2}\right)},
$$

where $u_{i} \equiv u\left(z_{i}\right)$. Using this identity, one can see that the uniformization (3.78), (3.79) converts equations (3.74), (3.75) into a single equation:

$$
\left(z_{1}^{-1}-z_{2}^{-1}\right) e^{\nabla\left(z_{1}\right) \nabla\left(z_{2}\right) F}=\frac{\theta_{1}\left(u\left(z_{1}\right)-u\left(z_{2}\right)\right)}{\theta_{4}\left(u\left(z_{1}\right)-u\left(z_{2}\right)\right)} .
$$

This equation should be compared with (3.45) which can be regarded as a limiting case of (3.81) as $\tau \rightarrow+\mathrm{i} \infty$. Note that the limit $z_{2} \rightarrow \infty$ in (3.81) gives the definition of the function $u(z)$ :

$$
e^{\partial_{t_{0}} \nabla(z) F}=z \frac{\theta_{1}(u(z))}{\theta_{4}(u(z))}
$$

(which is equivalent to the first formula in (3.78)). Next, the $z \rightarrow \infty$ limit in (3.82) yields

$$
R=e^{F_{00}}=\pi c_{1} \theta_{2}(0) \theta_{3}(0)
$$


hence we conclude that $c_{1}(\mathbf{t})=\gamma(\mathbf{t}) / \pi$.

Passing to logarithms and applying $\nabla\left(z_{3}\right)$ to the both sides of (3.81), we obtain another useful form of this equation. It is convenient to introduce the function

$$
S(u, \tau):=\log \frac{\theta_{1}(u, \tau)}{\theta_{4}(u, \tau)} .
$$

It has the following quasiperiodicity properties:

$$
S(u+1, \tau)=S(u, \tau)+i \pi, \quad S(u+\tau, \tau)=S(u, \tau) .
$$

Below we write simply $S(u, \tau)=S(u)$ but it is important that $S(u)$ depends on $\mathbf{t}$ not only through $u$ but also through $\tau$. In terms of this function, the equation (3.81) means that $\nabla\left(z_{3}\right) S\left(u\left(z_{1}\right)-u\left(z_{2}\right)\right)=\nabla\left(z_{3}\right) \nabla\left(z_{2}\right) \nabla\left(z_{1}\right) F$ is symmetric under permutations of $z_{1}, z_{2}, z_{3}$ :

$$
\nabla\left(z_{1}\right) S\left(u\left(z_{2}\right)-u\left(z_{3}\right)\right)=\nabla\left(z_{2}\right) S\left(u\left(z_{1}\right)-u\left(z_{3}\right)\right)=\nabla\left(z_{3}\right) S\left(u\left(z_{1}\right)-u\left(z_{2}\right)\right) .
$$

In particular, as $z_{3} \rightarrow \infty$ we get

$$
\nabla\left(z_{1}\right) S\left(u\left(z_{2}\right)\right)=\partial_{t_{0}} S\left(u\left(z_{1}\right)-u\left(z_{2}\right)\right)
$$

This is an elliptic extension of the first equation in (3.49). In the limit $z_{2} \rightarrow \infty$ equation (3.86) gives:

$$
\nabla(z) \log R=\partial_{t_{0}} S(u(z))
$$

Equations (3.85) are in fact equivalent to the dDKP hierarchy in the form (3.81). Indeed, it follows from (3.86) that $\nabla\left(z_{1}\right) S\left(u\left(z_{2}\right)\right)=\nabla\left(z_{2}\right) S\left(u\left(z_{1}\right)\right)$. Therefore, there exists a function $f=f(\mathbf{t})$ such that $S(u(z))=\nabla(z) f$. Substituting this into (3.86) and integrating with respect to $t_{0}$, we get

$$
\nabla\left(z_{1}\right) \nabla\left(z_{2}\right) F_{1}+\log \left(z_{1}^{-1}-z_{2}^{-1}\right)-S\left(u\left(z_{1}\right)-u\left(z_{2}\right)\right)=c\left(\mathbf{t}^{\prime}, z_{1}, z_{2}\right),
$$

where $\partial_{t_{0}} F_{1}=f, c\left(\mathbf{t}^{\prime}, z_{1}, z_{2}\right)=\sum_{n, m>0} c_{n m}\left(\mathbf{t}^{\prime}\right) z_{1}^{-n} z_{2}^{-m}$ is the integration constant and $\mathbf{t}^{\prime}=\left\{t_{1}, t_{2}, \ldots\right\}$. Applying $\nabla\left(z_{3}\right)$ to the both sides of this equation and using the symmetry of $\nabla\left(z_{3}\right) S\left(u\left(z_{1}\right)-u\left(z_{2}\right)\right)$ under the permutations of $z_{1}, z_{2}, z_{3}$ we conclude that $\nabla\left(z_{3}\right) c\left(\mathbf{t}^{\prime}, z_{1}, z_{2}\right)$ is also symmetric. Therefore, there exists a function $g$ such that $c\left(\mathbf{t}^{\prime}, z_{1}, z_{2}\right)=\nabla\left(z_{1}\right) \nabla\left(z_{2}\right) g$. Then $F=F_{1}-g$ satisfies (3.81). Thus we may say that the pair $u(z, \mathbf{t}), \tau(\mathbf{t})$ satisfying (3.85) is a solution to the dDKP hierarchy.

In order to connect the elliptic formulation with the algebraic one, we note that

$$
\log w(z)=-2 S(u(z)), \quad p(z)=c_{1} S^{\prime}(u(z)),
$$

where $S^{\prime}(u) \equiv \partial_{u} S(u)$. See [11] for details. 


\subsubsection{The reduction}

As in the previous cases, the one-variable reduction means that solutions of the hierarchy $u(z, \mathbf{t})$ and $\tau(\mathbf{t})$ depend on the times through a single variable $\lambda=\lambda(\mathbf{t}): u(z, \mathbf{t})=$ $u(z, \lambda(\mathbf{t})), \tau(\mathbf{t})=\tau(\lambda(\mathbf{t}))$. The function of two variables, $u(z, \lambda)$, can not be arbitrary. Our goal is to characterize the class of functions $u(z, \lambda), \tau(\lambda)$ that are consistent with the structure of the hierarchy and can be used for one-variable reductions. Below we obtain the consistency condition for one-variable reductions of the dDKP hierarchy in the elliptic form. The calculations are much more involved than in the cases of the dKP, dBKP and dToda hierarchies.

Applying the chain rule of differentiation to $S(u(z, \mathbf{t}) \mid \tau(\mathbf{t}))=S(u(z, \lambda(\mathbf{t})) \mid \tau(\lambda(\mathbf{t})))$, we get:

$$
\nabla\left(z_{1}\right) S\left(u\left(z_{2}\right)\right)=\left[\nabla\left(z_{1}\right) \lambda\right]\left(\partial_{\lambda} u\left(z_{2}\right) S^{\prime}\left(u\left(z_{2}\right)\right)+\partial_{\lambda} \tau \dot{S}\left(u\left(z_{2}\right)\right)\right),
$$

where the explicit dependence on $\tau$ is taken into account. Here and below

$$
\dot{S}(u)=\partial_{\tau} S(u, \tau)
$$

Next, we have, using (3.87):

$$
\begin{aligned}
& \nabla\left(z_{1}\right) \lambda=\frac{d \lambda}{d \log R} \nabla\left(z_{1}\right) \log R=\frac{d \lambda}{d \log R} \partial_{t_{0}} S\left(u\left(z_{1}\right)\right) \\
& =\frac{d \lambda}{d \log R} \partial_{t_{0}} \lambda\left(\partial_{\lambda} u\left(z_{1}\right) S^{\prime}\left(u\left(z_{1}\right)\right)+\partial_{\lambda} \tau \dot{S}\left(u\left(z_{1}\right)\right)\right) .
\end{aligned}
$$

In a similar way, we get:

$\partial_{t_{0}} S\left(u\left(z_{1}\right)-u\left(z_{2}\right)\right)=\partial_{t_{0}} \lambda\left[\left(\partial_{\lambda} u\left(z_{1}\right)-\partial_{\lambda} u\left(z_{2}\right)\right) S^{\prime}\left(u\left(z_{1}\right)-u\left(z_{2}\right)\right)+\partial_{\lambda} \tau \dot{S}\left(u\left(z_{1}\right)-u\left(z_{2}\right)\right)\right]$.

The formulas simplify a bit if we choose $\lambda=\tau$ which we set in what follows. Assuming that $\partial_{t_{0}} \tau \neq 0$ identically, we arrive at the following relation:

$$
\begin{gathered}
{\left[\partial_{\tau} u\left(z_{1}\right) S^{\prime}\left(u\left(z_{1}\right)\right)+\dot{S}\left(u\left(z_{1}\right)\right)\right]\left[\partial_{\tau} u\left(z_{2}\right) S^{\prime}\left(u\left(z_{2}\right)\right)+\dot{S}\left(u\left(z_{2}\right)\right)\right]} \\
=\frac{d \log R}{d \tau}\left[\left(\partial_{\tau} u\left(z_{1}\right)-\partial_{\tau} u\left(z_{2}\right)\right) S^{\prime}\left(u\left(z_{1}\right)-u\left(z_{2}\right)\right)+\dot{S}\left(u\left(z_{1}\right)-u\left(z_{2}\right)\right)\right] .
\end{gathered}
$$

To proceed further, we need to know $\dot{S}(u)$. It is given by equation (A11) in Appendix A. The proof can be found in [11], for the proof of similar formulae see [56, 57]. The key observation is that the substitutions

$$
\left\{\begin{array}{l}
4 \pi i \partial_{\tau} u=\zeta_{1}(\xi-u)+\zeta_{4}(\xi-u)-\zeta_{1}(\xi)-\zeta_{4}(\xi) \\
4 \pi i \partial_{\tau} \log R=\left(S^{\prime}(\xi)\right)^{2}
\end{array}\right.
$$

where $\xi$ is an arbitrary parameter, convert equation (3.90) into identity. It is a highly nontrivial statement. Here we omit the details which can be found in [11]. 
Therefore, using the identity $\zeta_{1}(u, \tau)+\zeta_{4}(u, \tau)=\zeta_{1}\left(u, \frac{\tau}{2}\right)$, we conclude that the function $u(z, \tau)$ is compatible with the infinite hierarchy if it satisfies the differential equation

$$
4 \pi i \partial_{\tau} u(z)=-\zeta_{1}\left(u(z)-\xi(\tau), \frac{\tau}{2}\right)-\zeta_{1}\left(\xi(\tau), \frac{\tau}{2}\right)
$$

where $\xi(\tau)$ can be arbitrary real-valued function of $\tau$. This is essentially the KomatuLöwner equation (2.32). The variables in $\$ 2.4$ correspond as

$$
\tau=2 \frac{\log q}{\pi i}, \quad u(z, \tau)=\frac{\log g(z, q)}{2 \pi i}, \quad \xi(\tau)=\frac{\log \Lambda(q)}{2 \pi i} .
$$

The driving function $\Lambda(q)=e^{2 \pi i \xi(\tau)}$, which encodes the shape of the slit in the Löwner theory, specifies the reduction.

Coming back to an arbitrary reduction variable $\lambda=\lambda(\tau)$, we obtain the equation

$$
4 \pi i \partial_{\lambda} u(z)=-\left[\zeta_{1}\left(u(z)-\xi(\lambda), \frac{\tau}{2}\right)+\zeta_{1}\left(\xi(\lambda), \frac{\tau}{2}\right)\right] \partial_{\lambda} \tau
$$

Equation (3.93) written for the inverse function to the $u(z), z(u)$, reads

$$
4 \pi i \partial_{\lambda} z(u)=-\left[\zeta_{1}\left(u-\xi(\lambda), \frac{\tau}{2}\right)+\zeta_{1}\left(\xi(\lambda), \frac{\tau}{2}\right)\right] \partial_{u} z(u) \partial_{\lambda} \tau
$$

One can also see that the second equation in (3.91),

$$
4 \pi i \partial_{\tau} \log R=\left(S^{\prime}(\xi(\tau))\right)^{2},
$$

emerges as the limiting case of (3.93) when $z \rightarrow \infty$. Using some identities for the function $S$, it is possible to show that for one-variable reductions the total $\tau$-derivative of $S(u(z))$ is given by

$$
4 \pi i \frac{d S(u(z))}{d \tau}=-S^{\prime}(\xi(\tau)) S^{\prime}(u(z)-\xi(\tau))
$$

Let us now pass to the system of reduced equations satisfied by $\lambda(\mathbf{t})$ and find their solution. Using (3.87), we can write:

$$
\nabla(z) \tau=\frac{\partial_{\tau} u(z) S^{\prime}(u(z))+\dot{S}(u(z))}{d \log R / d \tau} \partial_{t_{0}} \tau=\frac{d S(u(z)) / d \tau}{d \log R / d \tau} \partial_{t_{0}} \tau
$$

Substituting (3.95), (3.96) and passing to $\lambda=\lambda(\tau)$, we get:

$$
\nabla(z) \lambda=-\frac{S^{\prime}(u(z)-\xi(\lambda))}{S^{\prime}(\xi(\lambda))} \partial_{t_{0}} \lambda
$$

This is a generating equation for a hierarchy of equations of the hydrodynamic type. To write them explicitly, we use the expansion

$$
S^{\prime}(u-u(z))=S^{\prime}(u)+\sum_{k \geq 1} \frac{z^{-k}}{k} B_{k}^{\prime}(u)
$$


which defines the functions $B_{k}^{\prime}(u)=B_{k}^{\prime}(u, \tau)$. By analogy with the dispersionless KP case, one may think of them as elliptic analogues of the (derivatives of) Faber polynomials. In terms of the functions $B_{k}^{\prime}(u)$, the equations of the reduced hierarchy are as follows:

$$
\frac{\partial \lambda}{\partial t_{k}}=\phi_{k}(\xi(\lambda), \tau) \frac{\partial \lambda}{\partial t_{0}}, \quad \phi_{k}(\xi(\lambda), \tau):=\frac{B_{k}^{\prime}(\xi(\lambda), \tau)}{S^{\prime}(\xi(\lambda), \tau)}, \quad k \geq 1 .
$$

The common solution to these equations can be written in the hodograph form:

$$
t_{0}+\sum_{k=1}^{\infty} t_{k} \phi_{k}(\xi(\lambda), \tau)=\mathrm{R}(\lambda)
$$

where $\mathrm{R}(\lambda)$ is an arbitrary function of $\lambda$.

\section{Multivariable reductions}

\subsection{Multivariable reductions of the dKP hierarchy}

$N$-variable reduction of the dKP hierarchy means that the function $p(z)$ depends on the times t through $N$ functions $\lambda_{j}=\lambda_{j}(\mathbf{t})$, i.e., $p(z)=p(z, \mathbf{t})=p\left(z ; \lambda_{1}(\mathbf{t}), \ldots, \lambda_{N}(\mathbf{t})\right)$. Below we prove that solutions of a system of Löwner equations give solutions to the dKP hierarchy.

We consider the system of $N$ chordal Löwner equations of the form (3.18) which characterize the dependence of $p(z)=p\left(z ; \lambda_{1}, \ldots, \lambda_{N}\right)$ on the variables $\lambda_{j}$ :

$$
\frac{\partial p(z)}{\partial \lambda_{j}}=-\frac{1}{p(z)-\xi_{j}} \frac{\partial u}{\partial \lambda_{j}} .
$$

The real-valued driving functions $\xi_{j}$ are functions of $\lambda_{1}, \ldots, \lambda_{N}: \xi_{j}=\xi_{j}\left(\left\{\lambda_{i}\right\}\right)$. The compatibility condition of the system (4.1) is

$$
\frac{\partial}{\partial \lambda_{j}} \frac{\partial p(z)}{\partial \lambda_{k}}-\frac{\partial}{\partial \lambda_{k}} \frac{\partial p(z)}{\partial \lambda_{j}}=0
$$

A straightforward calculation (which uses the Löwner equations (4.1)) shows that it is equivalent to the following Gibbons-Tsarev system:

$$
\left\{\begin{array}{l}
\frac{\partial \xi_{j}}{\partial \lambda_{k}}=\frac{1}{\xi_{k}-\xi_{j}} \frac{\partial u}{\partial \lambda_{k}}, \\
\frac{\partial^{2} u}{\partial \lambda_{j} \partial \lambda_{k}}=\frac{2}{\left(\xi_{i}-\xi_{k}\right)^{2}} \frac{\partial u}{\partial \lambda_{j}} \frac{\partial u}{\partial \lambda_{k}}, \quad j \neq k .
\end{array}\right.
$$

Now assume that each $\lambda_{j}$ is a function of the time variables. Then for $p(z, \mathbf{t})=$ $p\left(z ; \lambda_{1}(\mathbf{t}), \ldots, \lambda_{N}(\mathbf{t})\right)$ we can rewrite equation (3.7) in the form

$$
-\sum_{j=1}^{N} D\left(z_{2}\right) \lambda_{j} \cdot \partial_{\lambda_{j}} p\left(z_{1}\right)=\sum_{j=1}^{N} \partial_{t_{1}} \lambda_{j} \cdot \partial_{\lambda_{j}} \log \left(p\left(z_{1}\right)-p\left(z_{2}\right)\right) .
$$


Using the Löwner equations (4.1), we find:

$$
\partial_{\lambda_{j}} \log \left(p\left(z_{1}\right)-p\left(z_{2}\right)\right)=\frac{\partial_{\lambda_{j}} u}{\left(p\left(z_{1}\right)-\xi_{j}\right)\left(p\left(z_{2}\right)-\xi_{j}\right)}
$$

and thus in order for (3.7) to be satisfied it is sufficient that the following equation holds:

$$
\sum_{j=1}^{N} D\left(z_{2}\right) \lambda_{j} \frac{\partial_{\lambda_{j}} u}{p\left(z_{1}\right)-\xi_{j}}=\sum_{j=1}^{N} \partial_{t_{1}} \lambda_{j} \frac{\partial_{\lambda_{j}} u}{\left(p\left(z_{1}\right)-\xi_{j}\right)\left(p\left(z_{2}\right)-\xi_{j}\right)}
$$

It is clear that if we introduce the dependence of the $\lambda_{j}$ 's on $\mathbf{t}$ by means of the relations

$$
D(z) \lambda_{j}=\frac{\partial_{t_{1}} \lambda_{j}}{p(z)-\xi_{j}}
$$

then equation (4.3) is satisfied identically.

Using definition of the Faber polynomials (3.11), one can see that equation (4.4) contains an infinite system of partial differential equations of hydrodynamic type:

$$
\frac{\partial \lambda_{j}}{\partial t_{k}}=\phi_{j, k}\left(\left\{\lambda_{i}\right\}\right) \frac{\partial \lambda_{j}}{\partial t_{1}}, \quad \phi_{j, k}=B_{k}^{\prime}\left(\xi_{j}\right), \quad k \geq 1, j=1, \ldots, N
$$

Note that $\phi_{j, 1}=B_{1}^{\prime}\left(\xi_{j}\right)=1$, so that equations (4.5) at $k=1$ become trivial identities. The generating function of $\phi_{j, k}\left(\left\{\lambda_{i}\right\}\right)$ is

$$
\sum_{k \geq 1} \phi_{j, k}\left(\left\{\lambda_{i}\right\}\right) \frac{z^{-k}}{k}=\frac{1}{p(z)-\xi_{j}}:=Q\left(p\left(z,\left\{\lambda_{i}\right\}\right), \xi_{j}\left(\left\{\lambda_{i}\right\}\right)\right) .
$$

We will show that the system (4.5) is consistent and can be solved by Tsarev's generalized hodograph method [12]. It can be directly verified that the compatibility condition of the system (4.5) is

$$
\frac{\partial_{\lambda_{j}} \phi_{i, n}}{\phi_{j, n}-\phi_{i, n}}=\frac{\partial_{\lambda_{j}} \phi_{i, n^{\prime}}}{\phi_{j, n^{\prime}}-\phi_{i, n^{\prime}}} \quad \text { for all } i \neq j, n, n^{\prime} .
$$

In other words the condition is that

$$
\Gamma_{i j}:=\frac{\partial_{\lambda_{j}} \phi_{i, n}}{\phi_{j, n}-\phi_{i, n}}
$$

does not depend on $n$. It is easy to see that this is equivalent to the $z$-independence of the ratio

$$
\Gamma_{i j}=\frac{\partial_{\lambda_{j}} Q\left(p(z), \xi_{i}\right)}{Q\left(p(z), \xi_{j}\right)-Q\left(p(z), \xi_{i}\right)}=\frac{\partial_{\lambda_{j}} u}{\left(\xi_{i}-\xi_{j}\right)^{2}},
$$

where $Q$ is the generating function (4.6). The proof of the second equality uses the Löwner equation (4.1) and the Gibbons-Tsarev equations (4.2).

Let $\mathrm{R}_{i}=\mathrm{R}_{i}\left(\left\{\lambda_{j}\right\}\right)(i=1, \ldots, N)$ satisfy the system of equations

$$
\frac{\partial \mathrm{R}_{i}}{\partial \lambda_{j}}=\Gamma_{i j}\left(\mathrm{R}_{j}-\mathrm{R}_{i}\right), \quad i, j=1, \ldots, N, \quad i \neq j,
$$


where $\Gamma_{i j}$ is defined in (4.9) (for $N=1$ this condition is void). We claim that the system (4.10) is compatible in the sense of Tsarev [12. To see this, we note that $\Gamma_{i j}$ (4.9) can be expressed as

$$
\Gamma_{i j}=\frac{1}{2} \frac{\partial}{\partial \lambda_{j}} \log g_{i}, \quad g_{i}=\frac{\partial u}{\partial \lambda_{i}}
$$

It then follows that

$$
\frac{\partial \Gamma_{i j}}{\partial \lambda_{k}}=\frac{\partial \Gamma_{i k}}{\partial \lambda_{j}}, \quad i \neq j \neq k,
$$

which are Tsarev's compatibility conditions. This means that the system (4.5) is semiHamiltonian. The main geometric object associated with a semi-Hamiltonian system is a diagonal metric. The quantities $g_{i}=g_{i i}$ are components of this metric while $\Gamma_{i j}=\Gamma_{i j}^{i}$ are the corresponding Christoffel symbols. In fact the metric $g_{i}$ is of Egorov type, i.e., it holds

$$
\frac{\partial g_{i}}{\partial \lambda_{k}}=\frac{\partial g_{k}}{\partial \lambda_{i}}
$$

(this follows from (4.11)).

Assume that $\mathrm{R}_{i}$ satisfy the system (4.10). Then the same argument as in the proof of Theorem 10 of Tsarev's paper [12] shows that if $\lambda_{i}(\mathbf{t})$ is defined implicitly by the hodograph relation

$$
t_{1}+\sum_{n \geq 2} \phi_{i, n}\left(\left\{\lambda_{j}\right\}\right) t_{n}=\mathrm{R}_{i}\left(\left\{\lambda_{j}\right\}\right)
$$

then $\lambda_{j}(\mathbf{t})$ satisfy (4.5).

We have found sufficient conditions for $N$-variable diagonal reductions of the dKP hierarchy. The reduction is given by a system of $N$ chordal Löwner equations (4.1) for a function $p\left(z, \lambda_{1}, \ldots, \lambda_{N}\right)$ supplemented by a diagonal system of hydrodynamic type (4.5) for the variables $\lambda_{j}, j=1, \ldots, N$.

\subsection{Multivariable reductions of the dBKP hierarchy}

We consider the system of $N$ quadrant Löwner equations of the form (3.34) which characterize the dependence of $p(z)=p\left(z ;\left\{\lambda_{j}\right\}\right)$ on the variables $\lambda_{j}$ :

$$
\frac{\partial p(z)}{\partial \lambda_{j}}=-\frac{p(z)}{p^{2}(z)-\xi_{j}^{2}} \frac{\partial u}{\partial \lambda_{j}} .
$$

The compatibility conditions of this system are given by the Gibbons-Tsarev equations which have the form [10]

$$
\left\{\begin{array}{l}
\frac{\partial \xi_{i}^{2}}{\partial \lambda_{j}}=\frac{2 \xi_{i}^{2}}{\xi_{j}^{2}-\xi_{i}^{2}} \frac{\partial u}{\partial \lambda_{j}} \\
\frac{\partial^{2} u}{\partial \lambda_{i} \partial \lambda_{j}}=\frac{2\left(\xi_{i}^{2}+\xi_{j}^{2}\right)}{\left(\xi_{i}^{2}-\xi_{j}^{2}\right)^{2}} \frac{\partial u}{\partial \lambda_{i}} \frac{\partial u}{\partial \lambda_{j}}, \quad i \neq j .
\end{array}\right.
$$


Now assume that each $\lambda_{j}$ is a function of $\mathbf{t}: p(z ; \mathbf{t})=p\left(z ;\left\{\lambda_{j}(\mathbf{t})\right\}\right)$. Then we can rewrite equation (3.29) of the $\mathrm{dBKP}$ hierarchy for $p(z ; \mathbf{t})$ in the form

$$
2 \sum_{j=1}^{N} D^{\circ}\left(z_{2}\right) \lambda_{j} \cdot \partial_{\lambda_{j}} p\left(z_{1}\right)=\sum_{j=1}^{N} \partial_{t_{1}} \lambda_{j} \cdot \partial_{\lambda_{j}} \log \frac{p\left(z_{1}\right)+p\left(z_{2}\right)}{p\left(z_{1}\right)-p\left(z_{2}\right)} .
$$

Using the quadrant Löwner equations (4.15), we find:

$$
\partial_{\lambda_{j}} \log \frac{p\left(z_{1}\right)+p\left(z_{2}\right)}{p\left(z_{1}\right)-p\left(z_{2}\right)}=-\frac{2 p\left(z_{1}\right) p\left(z_{2}\right) \partial_{\lambda_{j}} u}{\left(p^{2}\left(z_{1}\right)-\xi_{j}^{2}\right)\left(p^{2}\left(z_{2}\right)-\xi_{j}^{2}\right)},
$$

and thus in order for (3.29) to be satisfied it is sufficient that the following equation holds:

$$
\sum_{j=1}^{N} D^{\mathrm{o}}\left(z_{2}\right) \lambda_{j} \frac{p\left(z_{1}\right) \partial_{\lambda_{j}} u}{p^{2}\left(z_{1}\right)-\xi_{j}^{2}}=\sum_{j=1}^{N} \partial_{t_{1}} \lambda_{j} \frac{p\left(z_{1}\right) p\left(z_{2}\right) \partial_{\lambda_{j}} u}{\left(p^{2}\left(z_{1}\right)-\xi_{j}^{2}\right)\left(p^{2}\left(z_{2}\right)-\xi_{j}^{2}\right)} .
$$

It is clear that if we introduce the dependence of the $\lambda_{j}$ 's on $\mathbf{t}$ by means of the relations

$$
D^{\circ}(z) \lambda_{j}=\frac{p(z) \partial_{t_{1}} \lambda_{j}}{p^{2}(z)-\xi_{j}^{2}}
$$

then equation (4.17) is satisfied identically. Since

$$
\frac{p(z)}{p^{2}(z)-\xi_{j}^{2}}=\sum_{k \geq 1, \text { odd }} \frac{z^{-k}}{k} B_{k}^{\prime}\left(\xi_{j}\right)
$$

(see (3.32) ), we see that equation (4.18) contains an infinite system of partial differential equations of hydrodynamic type which have the same form as (4.5) with the only difference that $k$ is odd:

$$
\frac{\partial \lambda_{j}}{\partial t_{k}}=\phi_{j, k}\left(\left\{\lambda_{i}\right\}\right) \frac{\partial \lambda_{j}}{\partial t_{1}}, \quad \phi_{j, k}=B_{k}^{\prime}\left(\xi_{j}\right), \quad k=1,3,5, \ldots, \quad j=1, \ldots, N .
$$

The generating function of $\phi_{j, k}\left(\left\{\lambda_{i}\right\}\right)$ is

$$
\sum_{k \geq 1, \text { odd }} \phi_{j, k}\left(\left\{\lambda_{i}\right\}\right) \frac{z^{-k}}{k}=\frac{p(z)}{p^{2}(z)-\xi_{j}^{2}}:=Q\left(p\left(z,\left\{\lambda_{i}\right\}\right), \xi_{j}\left(\left\{\lambda_{i}\right\}\right)\right) .
$$

The compatibility condition of the system (4.19) has the same form (4.7) as before, which means that $\Gamma_{i j}$ given by (4.8) does not depend on $n$. Again, this is equivalent to the $z$-independence of

$$
\Gamma_{i j}=\frac{\partial_{\lambda_{j}} Q\left(p(z), \xi_{i}\right)}{Q\left(p(z), \xi_{j}\right)-Q\left(p(z), \xi_{i}\right)}=\frac{\xi_{i}^{2}+\xi_{j}^{2}}{\left(\xi_{i}^{2}-\xi_{j}^{2}\right)^{2}} \frac{\partial u}{\partial \lambda_{j}},
$$

where $Q$ is the generating function (4.20).

Let $R_{i}=R_{i}\left(\left\{\lambda_{j}\right\}\right)(i=1, \ldots, N)$ satisfy the system (4.10), where $\Gamma_{i j}$ is defined in (4.21). Tsarev's compatibility conditions (4.12) follow from (4.11) which remains of the same form and the same argument is used to show that if $\lambda_{i}(\mathbf{t})$ is defined implicitly by the hodograph relation

$$
t_{1}+\sum_{n \geq 3, \text { odd }} \phi_{i, n}\left(\left\{\lambda_{j}\right\}\right) t_{n}=R_{i}\left(\left\{\lambda_{j}\right\}\right),
$$

then $\lambda_{j}(\mathbf{t})$ satisfy (4.19). 


\subsection{Multivariable reductions of the dToda hierarchy}

We consider the system of $N$ radial Löwner equations of the form (3.64) which characterize the dependence of $w(z)=w\left(z ;\left\{\lambda_{j}\right\}\right)$ on the variables $\lambda_{j}$ :

$$
\frac{\partial \log w(z)}{\partial \lambda_{j}}=-\frac{w(z)+\eta_{j}}{w(z)-\eta_{j}} \frac{\partial \log r}{\partial \lambda_{j}}, \quad \eta_{j}=e^{i \xi_{j}} .
$$

The driving functions $\xi_{j}=\xi_{j}\left(\left\{\lambda_{i}\right\}\right)$ are real-valued. The compatibility conditions

$$
\frac{\partial}{\partial \lambda_{k}} \frac{\partial \log w(z)}{\partial \lambda_{j}}=\frac{\partial}{\partial \lambda_{j}} \frac{\partial \log w(z)}{\partial \lambda_{k}}
$$

of this system are given by the Gibbons-Tsarev equations:

$$
\left\{\begin{array}{l}
\frac{\partial \eta_{j}}{\partial \lambda_{k}}=\eta_{j} \frac{\eta_{k}+\eta_{j}}{\eta_{k}-\eta_{j}} \frac{\partial \log r}{\partial \lambda_{k}} \\
\frac{\partial^{2} \log r}{\partial \lambda_{j} \partial \lambda_{k}}=\frac{4 \eta_{j} \eta_{k}}{\left(\eta_{j}-\eta_{k}\right)^{2}} \frac{\partial \log r}{\partial \lambda_{j}} \frac{\partial \log r}{\partial \lambda_{k}}
\end{array}\right.
$$

or

$$
\left\{\begin{array}{l}
\frac{\partial \xi_{j}}{\partial \lambda_{k}}=\cot \left(\frac{1}{2}\left(\xi_{j}-\xi_{k}\right)\right) \frac{\partial \log r}{\partial \lambda_{k}} \\
\frac{\partial^{2} \log r}{\partial \lambda_{j} \partial \lambda_{k}}=-\frac{1}{\sin ^{2}\left(\frac{1}{2}\left(\xi_{j}-\xi_{k}\right)\right)} \frac{\partial \log r}{\partial \lambda_{j}} \frac{\partial \log r}{\partial \lambda_{k}}, \quad j \neq k .
\end{array}\right.
$$

Now assume that each $\lambda_{j}$ is a function of the times: $w(z ; \mathbf{t})=w\left(z ;\left\{\lambda_{j}(\mathbf{t})\right\}\right)$. Then we can rewrite equations (3.49) of the dToda hierarchy in the form

$$
\begin{aligned}
& -\sum_{j=1}^{N} D\left(z_{1}\right) \lambda_{j} \cdot \partial_{\lambda_{j}} \log w\left(z_{2}\right)=\sum_{j=1}^{N} \partial_{t_{0}} \lambda_{j}\left[\partial_{\lambda_{j}} \log \left(w\left(z_{1}\right)-w\left(z_{2}\right)\right)-\frac{1}{2} \partial_{\lambda_{j}} \log \frac{w\left(z_{1}\right)}{r}\right] \\
& \sum_{j=1}^{N} \bar{D}\left(\bar{z}_{1}\right) \lambda_{j} \cdot \partial_{\lambda_{j}} \log w\left(z_{2}\right)=\sum_{j=1}^{N} \partial_{t_{0}} \lambda_{j}\left[\partial_{\lambda_{j}} \log \left(\bar{w}\left(\bar{z}_{1}\right)-w^{-1}\left(z_{2}\right)\right)-\frac{1}{2} \partial_{\lambda_{j}} \log \frac{\bar{w}\left(\bar{z}_{1}\right)}{r}\right]
\end{aligned}
$$

Using the radial Löwner equations (4.23), we can rewrite these relations as

$$
\begin{gathered}
\sum_{j=1}^{N} D\left(z_{1}\right) \lambda_{j} \frac{w\left(z_{2}\right)+\eta_{j}}{w\left(z_{2}\right)-\eta_{j}} \partial_{\lambda_{j}} \log r=\sum_{j=1}^{N} \partial_{t_{0}} \lambda_{j} \frac{\eta_{j}\left(w\left(z_{2}\right)+\eta_{j}\right)}{\left(w\left(z_{1}\right)-\eta_{j}\right)\left(w\left(z_{2}\right)-\eta_{j}\right)} \partial_{\lambda_{j}} \log r, \\
-\sum_{j=1}^{N} \bar{D}\left(\bar{z}_{1}\right) \lambda_{j} \frac{w\left(z_{2}\right)+\eta_{j}}{w\left(z_{2}\right)-\eta_{j}} \partial_{\lambda_{j}} \log r=\sum_{j=1}^{N} \partial_{t_{0}} \lambda_{j} \frac{w\left(z_{2}\right)+\eta_{j}}{\left(1-\eta_{j} \bar{w}\left(\bar{z}_{1}\right)\right)\left(w\left(z_{2}\right)-\eta_{j}\right)} \partial_{\lambda_{j}} \log r .
\end{gathered}
$$

It is clear that if we introduce the dependence of the $\lambda_{j}$ 's on $\mathbf{t}, \overline{\mathbf{t}}$ by means of the relations

$$
\begin{aligned}
D(z) \lambda_{j} & =\frac{\eta_{j}}{w(z)-\eta_{j}} \partial_{t_{0}} \lambda_{j} \\
\bar{D}(\bar{z}) \lambda_{j} & =\frac{1}{\eta_{j} \bar{w}(\bar{z})-1} \partial_{t_{0}} \lambda_{j}
\end{aligned}
$$


then equations (4.26), (4.27) are satisfied identically. Note that the two equations (4.28) are complex conjugate to each other (recall that $\bar{\eta}_{j}=\eta_{j}^{-1}$ ).

As it follows from (3.67), equation (4.28) contains an infinite system of partial differential equations of hydrodynamic type:

$$
\frac{\partial \lambda_{j}}{\partial t_{k}}=\phi_{j, k}\left(\left\{\lambda_{i}\right\}\right) \frac{\partial \lambda_{j}}{\partial t_{0}}, \quad \phi_{j, k}=\eta_{j} A_{k}^{\prime}\left(\eta_{j}\right), \quad k \geq 1, \quad j=1, \ldots, N .
$$

The generating function of $\phi_{j, k}\left(\left\{\lambda_{i}\right\}\right)$ is

$$
\sum_{k \geq 1} \phi_{j, k}\left(\left\{\lambda_{i}\right\}\right) \frac{z^{-k}}{k}=\frac{\eta_{j}}{w(z)-\eta_{j}}:=Q\left(w\left(z,\left\{\lambda_{i}\right\}\right), \eta_{j}\left(\left\{\lambda_{i}\right\}\right)\right) .
$$

The compatibility condition of the system (4.29) has the same form as before. As a straightforward calculation shows,

$$
\Gamma_{i j}=\frac{\partial_{\lambda_{j}} Q\left(w(z), \eta_{i}\right)}{Q\left(w(z), \eta_{j}\right)-Q\left(w(z), \eta_{i}\right)}=\frac{2 \eta_{i} \eta_{j}}{\left(\eta_{i}-\eta_{j}\right)^{2}} \partial_{\lambda_{j}} \log r
$$

does not depend on $z$.

Let $R_{i}=R_{i}\left(\left\{\lambda_{j}\right\}\right)(i=1, \ldots, N)$ satisfy the system (4.10), where $\Gamma_{i j}$ is defined in (4.31). Tsarev's compatibility conditions (4.12) follow from the fact that

$$
\Gamma_{i j}=\frac{1}{2} \frac{\partial}{\partial \lambda_{j}} \log g_{i}, \quad g_{i}=\frac{\partial \log r}{\partial \lambda_{i}}
$$

which is easily checked using the Gibbons-Tsarev equation (4.24). Finally, the same argument as before is used to show that if $\lambda_{i}(\mathbf{t})$ is defined implicitly by the hodograph relation

$$
t_{0}+2 \operatorname{Re} \sum_{n \geq 1} \phi_{i, n}\left(\left\{\lambda_{j}\right\}\right) t_{n}=R_{i}\left(\left\{\lambda_{j}\right\}\right)
$$

then $\lambda_{j}(\mathbf{t})$ satisfy (4.29).

\subsection{Multivariable reductions of the dDKP hierarchy in the el- liptic form}

In this section we study diagonal $N$-variable reductions of the dDKP hierarchy in the elliptic form when the function $u=u(z)$ depends on the times $\mathbf{t}$ through $N$ real variables $\lambda_{j}: u(z ; \mathbf{t})=u\left(z ;\left\{\lambda_{j}(\mathbf{t})\right\}\right)$. Following [33], we prove that solutions of a system of elliptic Löwner equations give solutions to the dDKP hierarchy.

\subsubsection{The Gibbons-Tsarev equations and a system of hydrodynamic type}

The starting point is the system of $N$ elliptic Löwner equations which characterize the dependence of $u(z)$ on the variables $\lambda_{j}$ :

$$
\begin{aligned}
\frac{\partial u}{\partial \lambda_{j}} & =-\frac{1}{4 \pi \mathrm{i}}\left(\zeta_{1}\left(u-\xi_{j}, \tau\right)+\zeta_{4}\left(u-\xi_{j}, \tau\right)+\zeta_{1}\left(\xi_{j}, \tau\right)+\zeta_{4}\left(\xi_{j}, \tau\right)\right) \frac{\partial \tau}{\partial \lambda_{j}} \\
& =-\frac{1}{4 \pi \mathrm{i}}\left(\zeta_{1}\left(u-\xi_{j}, \tau^{\prime}\right)+\zeta_{1}\left(\xi_{j}, \tau^{\prime}\right)\right) \frac{\partial \tau}{\partial \lambda_{j}} .
\end{aligned}
$$


Here and below we abbreviate $\tau^{\prime}=\frac{\tau}{2}$. In (4.34), $u=u\left(z,\left\{\lambda_{i}\right\}\right)$ is a function of $z$ and real variables $\left\{\lambda_{i}\right\}=\left\{\lambda_{1}, \ldots, \lambda_{N}\right\}$ which are functions of the times. The real-valued driving functions are $\xi_{j}=\xi_{j}\left(\left\{\lambda_{i}\right\}\right)$.

The Gibbons-Tsarev system is the compatibility condition for the system of elliptic Löwner equations (4.34):

$$
\frac{\partial}{\partial \lambda_{j}} \frac{\partial u}{\partial \lambda_{k}}-\frac{\partial}{\partial \lambda_{k}} \frac{\partial u}{\partial \lambda_{j}}=0
$$

As is shown in [33], this compatibility condition is equivalent to the elliptic analogue of the Gibbons-Tsarev system:

$$
\begin{gathered}
\frac{\partial \xi_{k}}{\partial \lambda_{j}}=\frac{1}{4 \pi \mathrm{i}}\left(\zeta_{1}\left(\xi_{j}-\xi_{k}, \tau^{\prime}\right)-\zeta_{1}\left(\xi_{j}, \tau^{\prime}\right)\right) \frac{\partial \tau}{\partial \lambda_{j}}, \\
\frac{\partial^{2} \tau}{\partial \lambda_{k} \partial \lambda_{j}}=\frac{1}{2 \pi \mathrm{i}} \wp_{1}\left(\xi_{k}-\xi_{j}, \tau^{\prime}\right) \frac{\partial \tau}{\partial \lambda_{k}} \frac{\partial \tau}{\partial \lambda_{j}}
\end{gathered}
$$

for all $j=1, \ldots, N, j \neq k$. The system of equations (4.35), (4.36) is the elliptic analogue of the famous Gibbons-Tsarev system [3, 4]. They already appeared in the literature [58, 59, 60].

Let us calculate $\partial_{\lambda_{j}} S\left(u\left(z_{1}\right)-u\left(z_{2}\right)\right)$ :

$$
\partial_{\lambda_{j}} S\left(u_{1}-u_{2}\right)=S^{\prime}\left(u_{1}-u_{2}\right)\left(\frac{\partial u_{1}}{\partial \lambda_{j}}-\frac{\partial u_{2}}{\partial \lambda_{j}}\right)+\dot{S}\left(u_{1}-u_{2}\right) \frac{\partial \tau}{\partial \lambda_{j}}
$$

where we abbreviate $u_{i} \equiv u\left(z_{i}\right)$ and $\dot{S}(u)=\partial_{\tau} S(u, \tau)$. Plugging here the elliptic Löwner equations (4.34) and the formula (A11) for $\dot{S}(u)$, we have:

$$
\begin{gathered}
\partial_{\lambda_{j}} S\left(u_{1}-u_{2}\right)=\frac{1}{4 \pi \mathrm{i}} S^{\prime}\left(u_{1}-u_{2}\right)\left[-\zeta_{1}\left(u_{1}-\xi_{j}\right)-\zeta_{4}\left(u_{1}-\xi_{j}\right)+\zeta_{1}\left(u_{2}-\xi_{j}\right)+\zeta_{4}\left(u_{2}-\xi_{j}\right)\right. \\
\left.+2 \zeta_{2}\left(u_{1}-u_{2}\right)+\frac{\pi^{2} \theta_{4}^{4}(0, \tau)}{S^{\prime}\left(u_{1}-u_{2}\right)}\right] \frac{\partial \tau}{\partial \lambda_{j}}=\frac{1}{4 \pi \mathrm{i}} S^{\prime}\left(u_{1}-\xi_{j}\right) S^{\prime}\left(u_{2}-\xi_{j}\right) \frac{\partial \tau}{\partial \lambda_{j}} .
\end{gathered}
$$

Here we have used identity (A15) from [11]. In particular, tending $z_{2} \rightarrow \infty$, we get

$$
\partial_{\lambda_{j}} S(u(z))=-\frac{1}{4 \pi \mathrm{i}} S^{\prime}\left(\xi_{j}\right) S^{\prime}\left(u(z)-\xi_{j}\right) \frac{\partial \tau}{\partial \lambda_{j}}
$$

Let us construct a solution $u(z), \tau$ to the dDKP hierarchy which depends on times through the $\lambda_{i}$ 's: $u(z, \mathbf{t})=u\left(z,\left\{\lambda_{i}(\mathbf{t})\right\}\right), \tau(\mathbf{t})=\tau\left(\left\{\lambda_{i}(\mathbf{t})\right\}\right)$. It is an $N$-variable reduction of the hierarchy. If the reduction condition is imposed, main equation (3.86) reads

$$
\sum_{j=1}^{N} \nabla\left(z_{1}\right) \lambda_{j} \cdot \partial_{\lambda_{j}} S\left(u\left(z_{2}\right)\right)=\sum_{j=1}^{N} \partial_{t_{0}} \lambda_{j} \cdot \partial_{\lambda_{j}} S\left(u\left(z_{1}\right)-u\left(z_{2}\right)\right) .
$$

Plugging here equations (4.37), (4.38), we obtain:

$$
-\sum_{j=1}^{N} \nabla\left(z_{1}\right) \lambda_{j} \cdot S^{\prime}\left(\xi_{j}\right) S^{\prime}\left(u\left(z_{2}\right)-\xi_{j}\right) \frac{\partial \tau}{\partial \lambda_{j}}=\sum_{j=1}^{N} \partial_{t_{0}} \lambda_{j} \cdot S^{\prime}\left(u\left(z_{1}\right)-\xi_{j}\right) S^{\prime}\left(u\left(z_{2}\right)-\xi_{j}\right) \frac{\partial \tau}{\partial \lambda_{j}} .
$$


It is clear from (4.39) that if we introduce the dependence of the $\lambda_{j}$ 's on $\mathbf{t}$ by means of the relation

$$
\nabla(z) \lambda_{j}=-\frac{S^{\prime}\left(u(z)-\xi_{j}\right)}{S^{\prime}\left(\xi_{j}\right)} \frac{\partial \lambda_{j}}{\partial t_{0}}
$$

equation (4.39) is satisfied identically. It is easy to see that equations (3.85) are also satisfied. Indeed, using the reduction condition, we rewrite equation (3.85) as

$$
\sum_{j=1}^{N} \nabla\left(z_{3}\right) \lambda_{j} \cdot \partial_{\lambda_{j}} S\left(u\left(z_{1}\right)-u\left(z_{2}\right)\right) \text { is symmetric under permutations of } z_{1}, z_{2}, z_{3} \text {. }
$$

Plugging here (4.37), we have that $\sum_{j=1}^{N} \nabla\left(z_{3}\right) \lambda_{j} \cdot S^{\prime}\left(u\left(z_{1}\right)-\xi_{j}\right) S^{\prime}\left(u\left(z_{2}\right)-\xi_{j}\right)$ is symmetric under permutations of $z_{1}, z_{2}, z_{3}$ if $\nabla(z) \lambda_{j}$ is given by (4.40).

Equation (4.40) contains an infinite system of partial differential equations of hydrodynamic type. To write them out explicitly, we use the (derivatives of) elliptic Faber functions $B_{k}^{\prime}(u)$ introduced via the expansion (3.98). Then the system (4.40) reads

$$
\frac{\partial \lambda_{j}}{\partial t_{k}}=\phi_{j, k}\left(\left\{\lambda_{i}\right\}\right) \frac{\partial \lambda_{j}}{\partial t_{0}}, \quad \phi_{j, k}=\frac{B_{k}^{\prime}\left(\xi_{j}\right)}{S^{\prime}\left(\xi_{j}\right)}
$$

which is an infinite diagonal system of partial differential equations of hydrodynamic type. The $\lambda_{j}$ 's play the role of the Riemann invariants. The generating function of $\phi_{j, k}\left(\left\{\lambda_{i}\right\}\right)$ is

$$
\begin{gathered}
1+\sum_{k \geq 1} \phi_{j, k}\left(\left\{\lambda_{i}\right\}\right) \frac{z^{-k}}{k}:=Q\left(u\left(z,\left\{\lambda_{i}\right\}\right), \xi_{j}\left(\left\{\lambda_{i}\right\}\right), \tau\left(\left\{\lambda_{i}\right\}\right)\right), \\
Q(u, \xi, \tau)=\frac{S^{\prime}(\xi-u, \tau)}{S^{\prime}(\xi, \tau)} .
\end{gathered}
$$

It is convenient to put $\phi_{i, 0}=1$.

\subsubsection{Generalized hodograph method}

We have reduced the dDKP hierarchy to the system of elliptic Löwner equations and the auxiliary equations of hydrodynamic type

$$
\frac{\partial \lambda_{i}(\mathbf{t})}{\partial t_{n}}=\phi_{i, n}\left(\left\{\lambda_{j}(\mathbf{t})\right\}\right) \frac{\partial \lambda_{i}(\mathbf{t})}{\partial t_{0}}
$$

where $\phi_{i, n}$ are given by (4.41). This system is consistent and can be solved by Tsarev's generalized hodograph method [12] as were the previous cases (see sections 4.1, 4.2, 4.3).

It can be directly verified that the compatibility condition of the system (4.43) is

$$
\frac{\partial_{\lambda_{j}} \phi_{i, n}}{\phi_{j, n}-\phi_{i, n}}=\frac{\partial_{\lambda_{j}} \phi_{i, n^{\prime}}}{\phi_{j, n^{\prime}}-\phi_{i, n^{\prime}}} \quad \text { for all } i \neq j, n, n^{\prime}
$$


In other words the condition is that

$$
\Gamma_{i j}:=\frac{\partial_{\lambda_{j}} \phi_{i, n}}{\phi_{j, n}-\phi_{i, n}}
$$

does not depend on $n$, or, equivalently that

$$
\Gamma_{i j}=\frac{\partial_{\lambda_{j}} Q\left(u(z), \xi_{i}, \tau\right)}{Q\left(u(z), \xi_{j}, \tau\right)-Q\left(u(z), \xi_{i}, \tau\right)}
$$

does not depend on $z$, where $Q$ is the generating function (4.42). The independence of (4.45) of $z$ is proved in [33] by a direct cumbersome calculation and the coefficients $\Gamma_{i j}$ are found:

$$
\Gamma_{i j}=-\frac{1}{4 \pi \mathrm{i}} \frac{S^{\prime}\left(\xi_{j}\right)}{S^{\prime}\left(\xi_{i}\right)} S^{\prime \prime}\left(\xi_{i}-\xi_{j}\right) \frac{\partial \tau}{\partial \lambda_{j}} .
$$

Let $\mathrm{R}_{i}=\mathrm{R}_{i}\left(\left\{\lambda_{j}\right\}\right)(i=1, \ldots, N)$ satisfy the system of equations

$$
\frac{\partial \mathrm{R}_{i}}{\partial \lambda_{j}}=\Gamma_{i j}\left(\mathrm{R}_{j}-\mathrm{R}_{i}\right), \quad i, j=1, \ldots, N, \quad i \neq j
$$

where $\Gamma_{i j}$ is defined in (4.46) (for $N=1$ this condition is void). We claim that the system (4.47) is compatible in the sense of Tsarev [12]. To see this, we note that $\Gamma_{i j}$ (4.46) can be expressed as logarithmic derivative of a function:

$$
\Gamma_{i j}=\frac{1}{2} \frac{\partial}{\partial \lambda_{j}} \log g_{i}, \quad g_{i}=\frac{1}{4 \pi \mathrm{i}}\left(S^{\prime}\left(\xi_{i}\right)\right)^{2} \frac{\partial \tau}{\partial \lambda_{i}} .
$$

This is proved in [33]. It then follows that

$$
\frac{\partial \Gamma_{i j}}{\partial \lambda_{k}}=\frac{\partial \Gamma_{i k}}{\partial \lambda_{j}}, \quad i \neq j \neq k,
$$

which are Tsarev's compatibility conditions. This means that the system (4.43) is semiHamiltonian. The main geometric object associated with a semi-Hamiltonian system is a diagonal metric. The quantities $g_{i}=g_{i i}$ are components of this metric while $\Gamma_{i j}=\Gamma_{i j}^{i}$ are the corresponding Christoffel symbols. In fact the metric $g_{i}$ is of Egorov type, i.e., it holds

$$
\frac{\partial g_{i}}{\partial \lambda_{k}}=\frac{\partial g_{k}}{\partial \lambda_{i}}
$$

The proof is very simple:

$$
\frac{\partial g_{k}}{\partial \lambda_{i}}=g_{k} \frac{\partial \log g_{k}}{\partial \lambda_{i}}=2 g_{k} \Gamma_{k i}=\frac{1}{8 \pi^{2}} S^{\prime}\left(\xi_{i}\right) S^{\prime}\left(\xi_{k}\right) S^{\prime \prime}\left(\xi_{i}-\xi_{k}\right) \frac{\partial \tau}{\partial \lambda_{i}} \frac{\partial \tau}{\partial \lambda_{k}}
$$

from which we see that the right hand side is explicitly symmetric under the permutation of $i$ and $k$. (Here we have used (4.46) and (4.48).)

Assume that $\mathrm{R}_{i}$ satisfy the system (4.47). We claim that if $\lambda_{i}(\mathbf{t})$ is defined implicitly by the hodograph relation

$$
t_{0}+\sum_{n \geq 1} \phi_{i, n}\left(\left\{\lambda_{j}\right\}\right) t_{n}=\mathrm{R}_{i}\left(\left\{\lambda_{j}\right\}\right)
$$


then $\lambda_{j}(\mathbf{t})$ satisfy (4.43). The proof is almost the same as that of Theorem 10 of Tsarev's paper [12].

To conclude, we have found sufficient conditions for $N$-variable diagonal reductions of the dDKP hierarchy in the elliptic parametrization. The reduction is given by a system of $N$ elliptic Löwner equations (4.34) for a function $u\left(z, \lambda_{1}, \ldots, \lambda_{N}\right)$ supplemented by a diagonal system of hydrodynamic type (4.41) for the variables $\lambda_{j}, j=1, \ldots, N$. We have derived compatibility conditions for the elliptic Löwner equations which are elliptic analogues of the Gibbons-Tsarev equations and have proved solvability of the hydrodynamic type system by means of the generalized hodograph method.

\section{Acknowledgments}

We thank I. Hotta for valuable correspondence and K. Basarov for help in preparing figures. The work of A.Z. was supported in part by RFBR grant 18-01-00461. The work of T.T. has been funded within the framework of the HSE University Basic Research Program and the Russian Academic Excellence Project '5-100'.

\section{Appendix A: necessary functions and identities}

The Jacobi's theta-functions $\theta_{a}(u)=\theta_{a}(u, \tau), a=1,2,3,4$, are defined by the formulas

$$
\begin{aligned}
& \theta_{1}(u)=-\sum_{k \in \mathbb{Z}} \exp \left(\pi \mathrm{i} \tau\left(k+\frac{1}{2}\right)^{2}+2 \pi \mathrm{i}\left(u+\frac{1}{2}\right)\left(k+\frac{1}{2}\right)\right), \\
& \theta_{2}(u)=\sum_{k \in \mathbb{Z}} \exp \left(\pi \mathrm{i} \tau\left(k+\frac{1}{2}\right)^{2}+2 \pi \mathrm{i} u\left(k+\frac{1}{2}\right)\right), \\
& \theta_{3}(u)=\sum_{k \in \mathbb{Z}} \exp \left(\pi \mathrm{i} \tau k^{2}+2 \pi \mathrm{i} u k\right), \\
& \theta_{4}(u)=\sum_{k \in \mathbb{Z}} \exp \left(\pi \mathrm{i} \tau k^{2}+2 \pi \mathrm{i}\left(u+\frac{1}{2}\right) k\right),
\end{aligned}
$$

where $\tau$ is a complex parameter (the modular parameter) such that $\operatorname{Im} \tau>0$. The function $\theta_{1}(u)$ is odd, the other three functions are even. The infinite product representation for the $\theta_{1}(u)$ reads:

$$
\theta_{1}(u)=\mathrm{i} \exp \left(\frac{\mathrm{i} \pi \tau}{4}-\mathrm{i} \pi u\right) \prod_{k=1}^{\infty}\left(1-e^{2 \pi \mathrm{i} k \tau}\right)\left(1-e^{2 \pi \mathrm{i}((k-1) \tau+u)}\right)\left(1-e^{2 \pi \mathrm{i}(k \tau-u)}\right) .
$$

We also mention the identity

$$
\theta_{1}^{\prime}(0)=\pi \theta_{2}(0) \theta_{3}(0) \theta_{4}(0)
$$

Many useful identities for the theta functions can be found in [61].

All formulas for derivatives of elliptic functions with respect to the modular parameter follow from the "heat equation" satisfied by the theta-functions:

$$
4 \pi \mathrm{i} \partial_{\tau} \theta_{a}(u, \tau)=\partial_{u}^{2} \theta_{a}(u, \tau)
$$


In the main text we use the functions

$$
\zeta_{a}(x, \tau)=\frac{\partial}{\partial x} \log \theta_{a}(x, \tau), \quad \wp_{a}(x, \tau)=-\frac{\partial}{\partial x} \zeta_{a}(x, \tau), \quad a=1,2,3,4 .
$$

Obviously, $\zeta_{a}$ are odd functions. In particular, $\zeta_{1}(x, \tau)=\frac{1}{x}+O(x)$ as $x \rightarrow 0$ and $\zeta_{a}(0, \tau)=0$ for $a=2,3,4$. The functions $\zeta_{1}(x)=\zeta_{1}(x, \tau)$ and $\wp_{1}(x)=\wp(x, \tau)$ are up to some simple correction terms the Weierstrass $\zeta$ - and $\wp$-functions respectively.

From the infinite product representation (A2) follows the explicit form of $\zeta_{1}(u)$ :

$$
\zeta_{1}(u)=-\pi i \mathcal{K}_{e^{\pi i \tau}}\left(e^{2 \pi i u}\right), \quad \mathcal{K}_{q}(z):=\frac{1+z}{1-z}+2 \sum_{k=1}^{\infty} \frac{q^{2 k} z}{1-q^{2 k} z}+2 \sum_{k=1}^{\infty} \frac{q^{2 k}}{q^{2 k}-z} .
$$

The function $\mathcal{K}_{q}(z)$ is called Villat's function ((2.2) of [31]; cf. also [26]). Villat's kernel,

$$
\mathcal{K}_{q}(z, \zeta):=\mathcal{K}_{q}(z / \zeta)
$$

plays an important role in the Löwner theory of doubly connected domains ([24], [31], [26]).

Let us introduce the function

$$
S(x)=\log \frac{\theta_{1}(x, \tau)}{\theta_{4}(x, \tau)} .
$$

We denote $\partial_{x} S(x)=S^{\prime}(x), \partial_{x}^{2} S(x)=S^{\prime \prime}(x), \partial_{\tau} S(x)=\dot{S}(x)$. One can prove the following formulae (here and below $\tau^{\prime} \equiv \frac{\tau}{2}$ ):

$$
\begin{gathered}
S^{\prime}(x)=\pi \theta_{4}^{2}(0, \tau) \frac{\theta_{2}(x, \tau) \theta_{3}(x, \tau)}{\theta_{1}(x, \tau) \theta_{4}(x, \tau)}=\pi \theta_{3}\left(0, \tau^{\prime}\right) \theta_{4}\left(0, \tau^{\prime}\right) \frac{\theta_{2}\left(x, \tau^{\prime}\right)}{\theta_{1}\left(x, \tau^{\prime}\right)} \\
S^{\prime \prime}(x)=-\pi^{2} \theta_{2}^{2}(0, \tau) \theta_{3}^{2}(0, \tau) \theta_{4}^{3}(0, \tau) \frac{\theta_{4}(2 x, \tau)}{\theta_{1}^{2}(x, \tau) \theta_{4}^{2}(x, \tau)} \\
=-\pi^{2} \theta_{3}\left(0, \tau^{\prime}\right) \theta_{4}\left(0, \tau^{\prime}\right) \theta_{2}^{2}\left(0, \tau^{\prime}\right) \frac{\theta_{3}\left(x, \tau^{\prime}\right) \theta_{4}\left(x, \tau^{\prime}\right)}{\theta_{1}^{2}\left(x, \tau^{\prime}\right)} \\
2 \pi \mathrm{i} \dot{S}(x)=S^{\prime}(x) \zeta_{2}(x, \tau)+\frac{\pi^{2}}{2} \theta_{4}^{4}(0, \tau) \\
2 \pi \mathrm{i} \dot{S}^{\prime}(x)=S^{\prime \prime}(x) \zeta_{2}(x, \tau)-S^{\prime}(x) \wp_{2}(x, \tau) .
\end{gathered}
$$

It is clear from (A9), (A10) that $S^{\prime}(x+1)=S^{\prime}(x), S^{\prime}\left(x+\tau^{\prime}\right)=-S^{\prime}(x), S^{\prime \prime}(x+1)=S^{\prime \prime}(x)$, $S^{\prime \prime}\left(x+\tau^{\prime}\right)=-S^{\prime \prime}(x)$. Note that

$$
S^{\prime}(x) S^{\prime}\left(x+\frac{1}{2}\right)=-\pi^{2} \theta_{4}^{4}(0, \tau) .
$$

Clearly, $S^{\prime}(x)$ is an odd function. As $x \rightarrow 0$, we have:

$$
S^{\prime}(x)=\frac{1}{x}+O(x), \quad S^{\prime \prime}(x)=-\frac{1}{x^{2}}+O(1) .
$$




\section{References}

[1] M. Mineev-Weinstein, P. Wiegmann and A. Zabrodin, Integrable Structure of Interface Dynamics, Phys. Rev. Lett. 84 (2000) 5106-5109.

[2] P. Wiegmann and A. Zabrodin, Conformal maps and integrable hierarchies, Commun. Math. Phys. 213 (2000) 523-538.

[3] J. Gibbons and S. Tsarev, Reductions of the Benney equations, Phys. Lett. A211 (1996) 19-24.

[4] J. Gibbons and S. Tsarev, Conformal maps and reductions of the Benney equations, Phys. Lett. A258 (1999) 263-271.

[5] K. Löwner, Untersuchungen über schlichte konforme Abbildungen des Einheitskreises I, Math. Ann. 89 (1923) 103-121.

[6] M. Mañas, L. Martínez-Alonso, E. Medina, Reductions and hodograph solutions of the dispersionless KP hierarchy, J. Phys. A: Math. Gen. 35 (2002) 401-417.

[7] M. Mañas, S-functions, reductions and hodograph solutions of the $r$ th dispersionless modified KP and Dym hierarchies, J. Phys. A: Math. Gen. 37 (2004) 11191-11221.

[8] K. Takasaki and T. Takebe, Radial Löwner equation and dispersionless cmKP hierarchy. arXiv:nlin.SI/0601063

[9] T. Takebe, L.-P. Teo and A. Zabrodin, Löwner equation and dispersionless hierarchies, J. Phys. A: Math. Gen. 39 (2006) 11479-11501. arXiv:math/0605161

[10] T. Takebe, Dispersionless BKP hierarchy and quadrant Löwner equation, SIGMA 10 (2014) 023 (13 pp.). arXiv:1308.4584

[11] V. Akhmedova and A. Zabrodin, Dispersionless DKP hierarchy and elliptic Löwner equation, J. Phys. A: Math. Theor. 47 (2014) 392001. arXiv:1404.5135

[12] S.P. Tsarëv, The geometry of Hamiltonian systems of hydrodynamic type. The generalized hodograph method, Izv. Akad. Nauk SSSR Ser. Mat. 54 (1990) 1048-1068 (in Russian); Math. USSR-Izv. 37 (1991) 397-419 (English translation).

[13] P. Kufarev, V. Sobolev and L. Sporyševa, A certain method of investigation of extremal problems for functions that are univalent in the half-plane, Trudy Tomsk. Gos. Univ. Ser. Meh.-Mat. 200 (1968) 142-164 (in Russian).

[14] W. Kager and B. Nienhuis, Guide to Stochastic Loewner Evolution and its Applications, J. Stat. Phys. 115: 1149-1229 (2004).

[15] O. Schramm, Scaling limits of loop-erased random walks and uniform spanning trees, Israel J. Math. 118 (2000) 221-288.

[16] J. Cardy, SLE for theoretical physicist, Ann. Phys. 318 (2005) 81-118. 
[17] A. Del Monaco and P. Gumenyuk, Chordal Loewner equation, Complex analysis and dynamical systems VI, Part 2 (2016) 63-77. Contemp. Math., 667, Israel Math. Conf. Proc., Amer. Math. Soc., Providence, RI, 2016. math. CV/1302.0898.

[18] T. Takebe, Lectures on Dispersionless Integrable Hierarchies, Rikkyo Center of Mathematical Physics Lecture Note 2 (2014), http://id.nii.ac.jp/1062/00009024.

[19] Kamke E., Differentialgleichungen, Lösungsmethoden und Lösungen I, 6 Auflage, (in German) Mathematik und ihre Anwendungen in Physik und Technik. Reihe A. Bd. 18, Akademische Verlagsgesellschaft, Geest \& Portig K.-G., Leipzig 1959.

[20] M. Katori and S. Koshida, Conformal welding problem, flow line problem, and multiple Schramm-Loewner evolution, J. Math. Phys. 61, 083301 (2020), https://doi.org/10.1063/1.5145357

[21] P.L. Duren, Univalent functions, Grundlehren der Matematischen Wissenschaften 259, Springer-Verlag, New York, 1983.

[22] Y. Komatu, Über einen Satz von Herrn Löwner, Proc. Imp. Acad. Tokyo 16 (1940) 512-514 (in German).

[23] Y. Komatu, Theory of confomal mappings vol.I, Kyoritsu-shuppan, Japan, 1944 (in Japanese).

[24] Y. Komatu, Untersuchungen über konforme Abbildung von zweifach zusammenhängenden Gebieten, Proc. of the Physico-Mathematical Society of Japan 25 (1943) 1-42 (in German). (Avaliable via J-Stage: https://www.jstage.jst.go.jp).

[25] Y. Komatu, Theory of confomal mappings vol.II, Kyoritsu-shuppan, Japan, 1949 (in Japanese).

[26] G. Goluzin, On parametric representation of functions univalent in an annulus, Matem. Sbornik 29 (1951) 469-476 (in Russian).

[27] I. A. Alexandrov, Parametric Continuations in the Theory of Univalent Functions, Nauka, Moscow, 1976 (in Russian).

[28] M. D. Contreras, S. Diaz-Madrigal and P. Gumenyuk, Loewner Theory in annulus I: evolution families and differential equations, Trans. Amer. Math. Soc. 365 (2013) 2505-2543.

[29] M. D. Contreras, S. Diaz-Madrigal and P. Gumenyuk, Loewner Theory in annulus II: Loewner chains, Anal. Math. Phys. 1 (2011) 351-385.

[30] F. Bracci, M. D. Contreras, S. Diaz-Madrigal and A. Vasil'ev, Classical and stochastic Löwner-Kufarev equations, Harmonic and Complex Analysis and Applications, Birkhäuser-Verlag, 2013, pp. 39-134.

[31] M. Fukushima, H. Kaneko, On Villat's kernels and BMD Schwarz kernels in Komatu-Loewner equations, in: Stochastic Analysis and Applications 2014, Oxford, 2013, (eds. D. Crisan, B. Hambly and T. Zariphopoulou), Springer Proc. Math. Stat., 100, Springer, 2014, pp.327-348. 
[32] V. Akhmedova and A. Zabrodin, Elliptic parametrization of Pfaff integrable hierarchies in the zero dispersion limit, Theor. Math. Phys. 185 (2015) 410-422. arXiv:1412.8435

[33] V. Akhmedova, T. Takebe and A. Zabrodin, Multi-variable reductions of the dispersionless DKP hierarchy, Journal of Physics A: Math. Theor. 50 (2017) 485204, arXiv:1707.01528,

[34] K. Takasaki and T. Takebe, Integrable hierarchies and dispersionless limit, Rev. Math. Phys. 7 (1995) 743-808. arXiv:hep-th/9405096

[35] L.-P. Teo, Analytic functions and integrable hierarchies - characterization of tau functions, Letters in Mathematical Physics 64 (2003) 75-92, arXiv:hep-th/0305005.

[36] A. Zabrodin, Growth of fat slits and dispersionless KP hierarchy, J. Phys. A: Math. Theor. 42 (2009) 085206 (23pp), arXiv: 0811.4054.

[37] L.V. Bogdanov and B.G. Konopelchenko, On dispersionless BKP hierarchy and its reductions, J. Nonlinear Math. Phys. 12, suppl. 1 (2005) 64-75, arXiv:nlin.SI/0411046.

[38] Y.-T. Chen and M.-H. Tu, A note on the dispersionless BKP hierarchy, J. Phys. A: Math. Gen. 39 (2006) 7641-7655.

[39] K. Takasaki, Dispersionless Hirota equations of two-component BKP hierarchy, SIGMA 2 (2006) 057.

[40] A. Zabrodin, Dispersionless limit of Hirota equations in some problems of complex analysis, Teor. Mat. Fys. 129 (2001) 239-257 (English translation: Theor. Math. Phys. 129 (2001) 1511-1525).

[41] A. Zabrodin, Growth processes related to the dispersionless Lax equations, Physica D235 (2007) 101-108, arXiv:math-ph/0609023.

[42] M. Jimbo and T. Miwa, Soliton equations and infinite dimensional Lie algebras, Publ. RIMS, Kyoto University 19 (1983) 943-1001.

[43] R. Hirota and Y. Ohta, Hierarchies of coupled soliton equations I, J. Phys. Soc. Japan 60 (1991) 798-809.

[44] M. Adler, E. Horozov and P. van Moerbeke, The Pfaff lattice and skew-orthogonal polynomials, Int. Math. Res. Notices 1999 (1999), no 11, 569-588.

[45] M. Adler, T. Shiota and P. van Moerbeke, Pfaff $\tau$-functions, Math. Ann. 322 (2002) 423-476.

[46] S. Kakei, Orthogonal and symplectic matrix integrals and coupled KP hierarchy, J. Phys. Soc. Japan 99 (1999) 2875-2877.

[47] S. Isojima, R. Willox and J. Satsuma, On various solutions of the coupled KP equation, J. Phys. A: Math. Gen. 35 (2002) 6893-6909. 
[48] R. Willox, On a generalized Tzitzeica equation, Glasgow Math. J. 47A (2005) 221231.

[49] Y. Kodama and K.-I. Maruno, N-soliton solutions to the DKP hierarchy and the Weyl group actions, J. Phys. A: Math. Gen. 39 (2006) 4063-4086.

[50] Y. Kodama and V. Pierce, Combinatorics of dispersionless integrable systems and universality in random matrix theory, Commun. Math. Phys. 292 (2009) 529-568.

[51] M. Adler, V. Kuznetsov and P. van Moerbeke, Rational solutions to the Pfaff lattice and Jack polynomials, Ergodic Theory Dynam. Systems 22 (2002) 1365-1405.

[52] J. van de Leur, Matrix integrals and the geometry of spinors, J. Nonlinear Math. Phys. 8 (2001) 288-310.

[53] A. Orlov, Deformed Ginibre ensembles and integrable systems, Phys. Lett. A 378 (2014) 319-328. arXiv:1210.1123

[54] K. Takasaki, Differential Fay identities and auxiliary linear problem of integrable hiearchies, Advanced Studies in Pure Mathematics 61 (2011) 387-441. arXiv:0710.5356

[55] K. Takasaki, Auxiliary linear problem, difference Fay identities and dispersionless limit of Pfaff-Toda hierarchy, SIGMA 5 (2009) 109. arXiv:0908.3569

[56] K. Takasaki, Painlevé-Calogero correspondence revisited, J. Math. Phys. 42 (2001) 1443-1473. arXiv:math/0004118

[57] A. Zabrodin and A. Zotov, Quantum Painlevé-Calogero correspondence for Painleve VI, J. Math. Phys. 53 (2012) 073508.

[58] A. Odesskii and V. Sokolov, Systems of Gibbons-Tsarev type and integrable 3dimensional models. arXiv:nlin/0906.3509

[59] A. Odesskii and V. Sokolov, Integrable $(2+1)$-dimensional systems of hydrodynamic type, Theor. Math. Phys. 163 (2010) 549-586. arXiv:nlin/1009.2778

[60] A. Odesskii and V. Sokolov, Integrable elliptic pseudopotentials, Theor. Math. Phys., 161 (2009) 1340-1352. arXiv:0810.3879

[61] S. Kharchev and A. Zabrodin, Theta vocabulary I, Journal of Geometry and Physics, 94 (2015) 19-31. arXiv:1502.04603 